\title{
Inertial Attitude Control of a Bat-like Morphing-wing Micro Air Vehicle
}

\author{
J. Colorado ${ }^{1}$, A. Barrientos ${ }^{1}$, and C. Rossi $^{1}$ \\ ${ }^{1}$ Centre for Automation and Robotics, Universidad Politécnica de Madrid and CSIC, 28006, \\ Madrid, Spain. \\ E-mail: jd.colorado@upm.es
}

\begin{abstract}
.
This article presents a novel bat-like micro air vehicle inspired by the morphing-wing mechanism of bats. The goal of this paper is twofold. Firstly, a modelling framework is introduced for analysing how the robot should maneuver by means of changing wing morphology. This allows the definition of requirements for achieving forward and turning flight according to the kinematics of the wing modulation. Secondly, an attitude controller named backstepping+DAF is proposed. Motivated by the biological fact about the influence of wing inertia on the production of body accelerations, the attitude control law incorporates wing inertia information to produce desired roll $(\phi)$ and pitch $(\theta)$ acceleration commands (DAF function). This novel control approach is aimed at incrementing net body forces $\left(F_{n e t}\right)$ that generate propulsion. Simulations and wind-tunnel experimental results have shown an increase about $23 \%$ in net body force production during the wingbeat cycle when the wings are modulated using the DAF function as a part of the backstepping control law. Results also confirm accurate attitude tracking in spite of high external disturbances generated by aerodynamic loads at airspeeds up to $5 \mathrm{~ms}^{-1}$.
\end{abstract}




\section{Introduction}

In recent years, the concept of morphing Micro Air Vehicles (MAVs) has gained interest [1], [2], [3]. The possibility of having actuated wings has allowed the design of new mechanisms that improve over classical fixed/rotary-wings MAV flight performance. As a result, different morphing-wing concepts and materials have emerged together with control methodologies that allow for accurate wing-actuation [4], [5], [6], [7], [8], [9].

The concept of morphing-wings comes from nature [10], [11]. Recently, the biological community has demonstrated a special interest in understanding and quantifying bat flight motivated by the sophistication of their flight apparatus [12], [13], [14]. Bats are animals that posses high maneuvering capabilities. Their wings contain dozens of articulations that allow the animal to perform aggressive maneuvers by means of controlling the wing shape during flight (morphing-wings). There is no other flying creature in nature with this level of wing dexterity and there is biological evidence that the inertial forces produced by the wings have a key role in the attitude movements of the animal [15], [16], [17], [18]. This can inspire the design of highly articulated morphing-wing micro air vehicles (not necessarily bat-like) with a significant wing-to-body mass ratio.

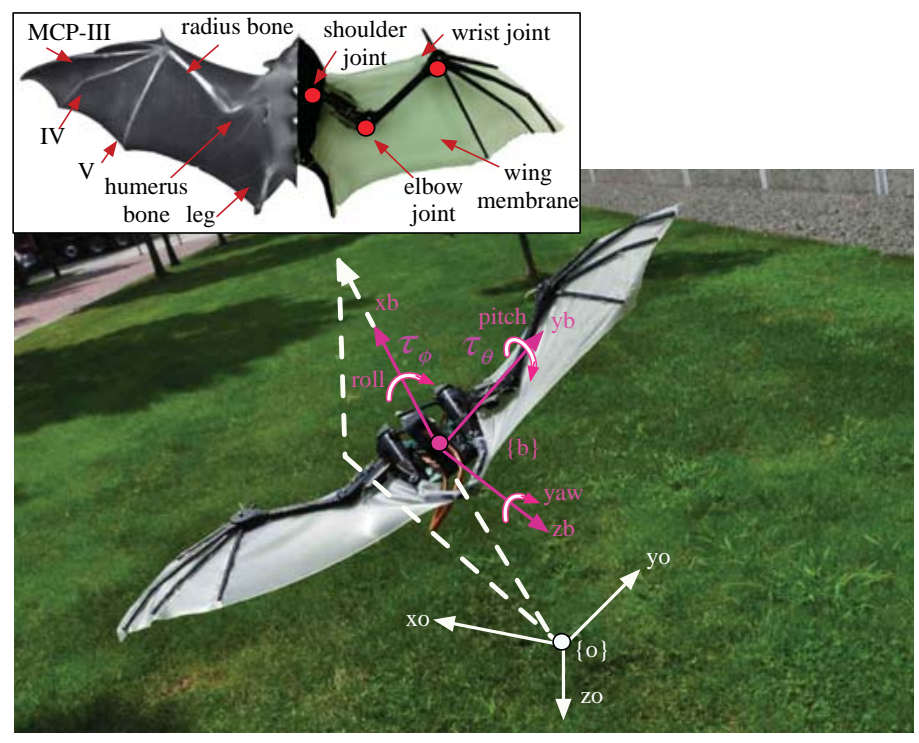

Figure 1. Bat-like robot main frame of references and wing components.

Attempting to mimic the mechanics of bat flight seems to have great potential to improve the maneuverability of current micro aerial vehicles. To the best of the authors' knowledge, there are not morphing-wing MAVs with highly articulated wings inspired by the biomechanics of bats. Motivated by this fact, prior work in [19] introduced the biological inspiration for the development of a novel bat-like robot with actuated morphing-wings. Shape Memory Alloys (SMAs) were used as artificial muscles for actuating the elbow and wrist joints. Experiments were conducted to assess the performance of the SMA muscles, aimed at developing a morphing-wing controller that allows the robot to change wing 
morphology. Figure 1 shows the bat-like MAV. The overall mass of the skeleton, electronics and battery is $125 \mathrm{~g}$ and the wingspan is $53 \mathrm{~cm}$ when wings are fully extended. Each wing of the robot has six degrees of freedom (dof): 2-dof at shoulder, 1-dof at elbow, and 3-dof at wrist joint. The body frame $\{b\}$ is a 6-dof floating body. Rotations about the body-frame $\{b\}$ $x_{b}, y_{b}, z_{b}$ axes are designated roll, pitch and yaw following aerodynamic conventions. Frame $\{o\}$ is the inertial frame.

This paper focuses on attitude control, analysing how the bat-like MAV can maneuver by means of changing the wing shape based on the morphing-wing control mechanism presented in [19]. In bats, there is biological evidence that the inertial forces produced by the wings have a significant contribution to the attitude movements of the animal, even more significant than aerodynamic forces [13], [14]. In fact, bats perform complex aerial rotations by solely modulating wing inertia [20], [21]. This means bats are able to change the orientation of the body during flight without relying on aerodynamic forces and instead by changing the mass distribution of its body and wings. Inertial forces are likely to be significant in bats because the mass of the wings comprises a significant portion of total body mass, ranging from $11 \%$ to $33 \%$, and because wings undergo large accelerations [22].

Taking into account the effects of wing inertia within the control law is a key factor for the design of an attitude controller of the proposed robot. The approach followed is aimed at defining proper references that drive the modulation of the wings' shape in such a way as to increment inertial forces that generate propulsion. To achieve this, an attitude control strategy based on backstepping plus a Desired Angular acceleration Function (DAF) to produce desired roll and pitch angular accelerations is used.

The DAF function contains wing inertia information that is provided by the inverse and forward dynamics computation of the Equations of Motion (EoM) of the robot. Spatial Newton-Euler formalism [23] is used to express the EoM and therefore theDAF function. This enhanced controller is called backstepping+DAF. The contributions of this paper are as follows:

- It describes a novel bat-like MAV with highly articulated wings that can be controlled. Taking advantage on this modelling morphing-wing mechanism, it is evaluated how the robot can maneuver by means of modulating wing inertia, without the need for any extra mechanisms such as ailerons or rudders.

- It proposes a nonlinear control approach, called backstepping+DAF, aimed at improving the attitude response of the MAV. Such enhancement is based on the assumption (motivated by the cited biological studies) that bats efficiently generate forward thrust by means of inertia wing modulation, taking advantage of relevant wing-to-body mass ratio.

The paper is organised as follows: Section 2 briefly describes the methodologies used for the description of the modelling framework. It describes kinematics, dynamics, wingactuation, flight control and aerodynamics. Symbols are listed and defined in Table 1. Section 3 presents the modelling framework. It shows quantification related to the importance of wing inertia for maneuvering. Section 4 introduces the proposed attitude controller. It 
Table 1. List of Symbols

\begin{tabular}{|c|c|}
\hline Symbol & Description \\
\hline$a$ & distance from axis $z_{i-1}$ to $z_{i}$ measured along $x_{i-1}$ \\
\hline$A_{b}$ & extended wing area \\
\hline AoA & angle of attack \\
\hline$B$ & extended wing length \\
\hline$C_{L}, C_{D}$ & lift and drag coefficients \\
\hline$d$ & distance from axis $x_{i-1}$ to $x_{i}$ measured along $z_{i}$ \\
\hline$f$ & wingbeat frequency \\
\hline$i$ & subscript that indicates a joint frame \\
\hline$f_{i}$ & force of body $i$ with respect to joint frame $\{i\}$ \\
\hline$F_{i}$ & spatial force of body $i$ with respect to joint frame $\{i\}$ \\
\hline$F_{L}, F_{D}$ & lift and drag forces measured about body frame $\{b\}$ \\
\hline$F_{n e t}$ & net forces measured about body frame $\{b\}$ \\
\hline$F_{T}$ & spatial forces with respect to the base frame $\{0\}$ \\
\hline$H$ & projection onto the axis of motion \\
\hline$I_{x x}, I_{y y}, I_{z z}$ & moments of inertia of body $i$ with respect to frame $\{\mathrm{cm}\}$ \\
\hline$I_{i, c m}$ & spatial inertia of body $i$ with respect to frame $\{\mathrm{cm}\}$ \\
\hline$I_{b}$ & spatial body inertia with respect to the body frame $\{b\}$ \\
\hline$I_{\text {sma }}$ & electrical current input to SMA actuators \\
\hline$J_{i, \mathrm{~cm}}$ & inertial tensor of body $i$ with respect to frame $\{\mathrm{cm}\}$ \\
\hline$l_{h}, l_{r}$ & humerus and radius bones length \\
\hline$L_{i, c m}$ & spatial inertial moment of body $i$ with respect to frame $\{\mathrm{cm}\}$ \\
\hline$M_{b}$ & body mass \\
\hline$m_{w}$ & wing mass (shoulder, radius, humerus, digits and membrane) \\
\hline$n_{i}$ & torque of body $i$ with respect to joint frame $\{i\}$ \\
\hline$p_{i, i+1}$ & $3 \times 1$ position vector that joins frame $\{i\}$ to $\{i+1\}$ \\
\hline$\tilde{p}_{i, i+1}$ & skew symmetric matrix of vector cross product of $p_{i, i+1}$ \\
\hline$P_{i, i+1}$ & spatial translation from joint frame $\{i\}$ to $\{i+1\}$ \\
\hline$P_{\text {sma }}$ & input heating power to SMA actuators \\
\hline$q_{i}, \dot{q}_{i}, \ddot{q}_{i}$ & joint positions, velocities and accelerations of body $i$ with respect to $\{i\}$ \\
\hline$r_{i+1, i}$ & $3 \times 3$ basic rotation matrix that projects frame $\{i+1\}$ onto frame $\{i\}$ \\
\hline$R_{i+1, i}$ & spatial rotation from joint frame $\{i+1\}$ to $\{i\}$ \\
\hline$R_{\text {sma }}$ & electrical resistance of SMA actuators \\
\hline$s_{i, c m}$ & $3 \times 1$ position vector that joins frame $\{i\}$ to $\{\mathrm{cm}\}$ \\
\hline$\tilde{s}_{i, c m}$ & skew symmetric matrix of vector cross product of $s_{i, \mathrm{~cm}}$ \\
\hline$S_{i, c m}$ & spatial translation from joint frame $\{i\}$ to $\{\mathrm{cm}\}$ \\
\hline$T_{i+1, i}$ & $4 \times 4$ homogeneous transformation matrix that relates frame $\{i+1\}$ with $\{i\}$ \\
\hline$u$ & control inputs \\
\hline$U$ & $3 \times 3$ identity operator \\
\hline$v_{i}, \dot{v}_{i}$ & linear velocity and acceleration of body $i$ with respect to joint frame $\{i\}$ \\
\hline$V_{i}, \dot{V}_{i}$ & spatial velocity and acceleration of body $i$ with respect to joint frame $\{i\}$ \\
\hline$\dot{V}_{b}$ & six-dimensional body accelerations with respect to the body frame $\{b\}$ \\
\hline$\alpha$ & is the angle from axis $z_{i-1}$ to $z_{i}$ measured about $x_{i-1}$ \\
\hline$c, \lambda$ & backstepping+DAF control gains \\
\hline$\phi, \theta$ & roll and pitch angles measured about body-frame $\{b\}$ \\
\hline$\ddot{\phi}^{d}, \ddot{\theta}^{d}$ & desired angular acceleration functions (DAF) \\
\hline$\tau_{\phi}, \tau_{\theta}$ & rolling and pitching torques measured about body-frame $\{b\}$ \\
\hline$\omega_{i}, \dot{\omega}_{i}$ & angular velocity and acceleration of body $i$ with respect to joint frame $\{i\}$ \\
\hline
\end{tabular}

details how to incorporate wing inertia information within the backstepping+DAF control law. Section 5 presents the control results. Wind-tunnel experiments are carried out for: i) evaluating the accuracy of the controller for tracking pitch and roll references under the presence of external disturbances caused by aerodynamic loads at airspeeds up to $5 m s^{-1}$, ii) demonstrating the assumption of incrementing net body forces thanks to the wing modulation driven by the backstepping+DAF controller. Section 6 concludes about the potential of the proposed methodologies toward achieving the first bat-like MAV capable of autonomous high maneuverable flight. 


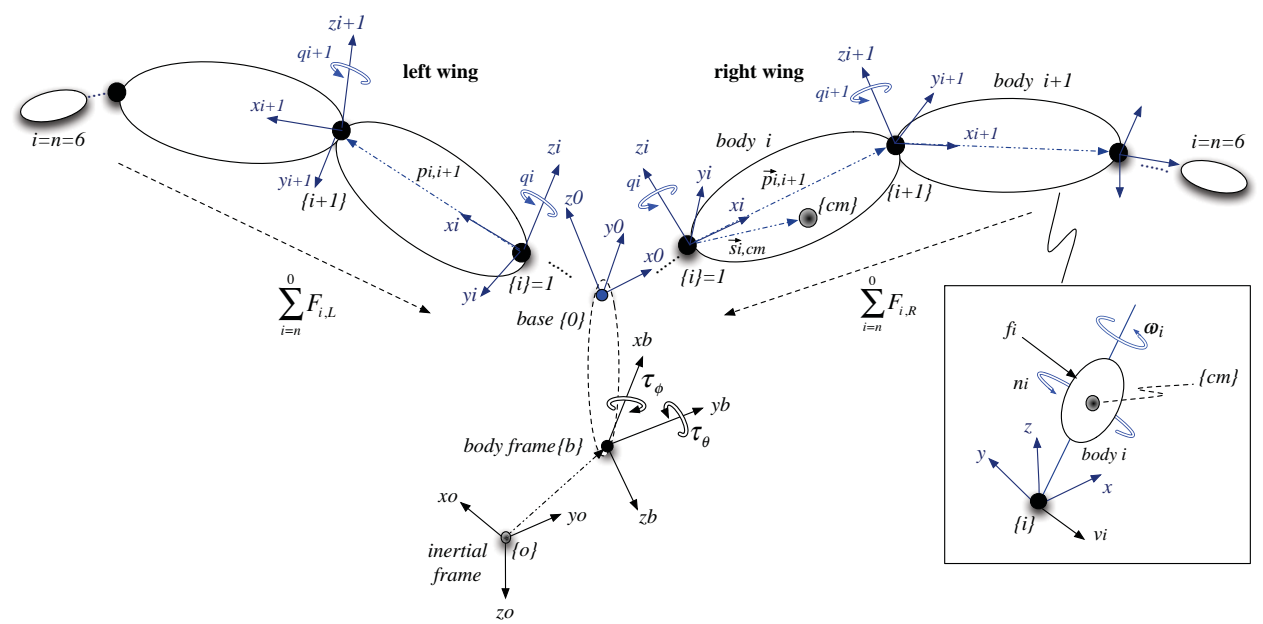

Figure 2. Rigid multi-body serial chain that composes each wing. Spatial forces of each body contain both linear $f_{i}$ and angular $n_{i}$ force components stacked into a sixdimensional vector $F_{i}$. These forces are propagated from the wingtip $\{i\}=n$ to the base frame $\{0\}$. Subscripts $R, L$ denote right and left wing respectively. The resultant spatial force $\left(F_{T}\right)$ acting on the base frame $\{0\}$ is the sum of spatial forces generated by both wings. The inset shows the velocity of a rigid body $i$ expressed in terms of $\omega_{i}$ and $v_{i}$, and the force acting on a rigid body $i$ expressed in terms of $f_{i}$ and $n_{i}$.

\section{Methods}

This section briefly introduces the methodologies used for the description of: i) kinematics, ii) dynamics, iii) wing-actuation, and iv) flight control and aerodynamics.

\subsection{Kinematics}

The bat robot is kinematically represented as two serial chains of rigid bodies (wings) connected to a base (body). The kinematics frames are described in figure 2. Morphological parameters of the bat-like MAV, shown in figure 1, have been inspired by the morphology of Pteropus poliocephalu specimen [24]. In this species, wings' mass accounts for $31 \%$ of the total mass, while in the robot wings' mass accounts for $37 \%$ of the total mass. Table 5 in Section 5 details morphological data of the specimen and the bat robot.

\section{Wing-frames}

Each wing, i.e., frames from $\{1\}$ to $\{6\}$ is treated as a serial chain of rigid bodies connected to a base frame $\{0\}$. Frames $\{0\}$ to $\{6\}$ have been placed following modified DenavitHartenberg (DH) convention [25]. Figure 2 details the DH frames whereas Table 2 describes the DH parameters. The base frame $\{0\}$ is a rotated body frame $\{b\}$ that allows the axis $x_{0}$ to point laterally toward the right wing. Axes $z_{1}$ to $z_{6}$ are aligned with the six joint axes such that $z_{i}$ is aligned with the axis of joint frame $\{i\}$. Axis $x_{i}$ is the common perpendicular between $z_{i}$ and $z_{i+1}$, directed from $z_{i}$ to $z_{i+1}$. The joint angles $q_{i}$ are defined from axis $x_{i-1}$ to $x_{i}$ measured 
Table 2. Modified Denavit-Hartenberg parameters for each-wing.

\begin{tabular}{cccccc}
\hline \hline Joint & Frame & $\alpha$ & $a$ & $q$ & $d$ \\
\hline Shoulder & $\{1\}$ & $\pi / 2$ & $l_{m} / 2=0.035 m$ & $q_{1}$ & 0 \\
Shoulder & $\{2\}$ & $-\pi / 2$ & 0 & $q_{2}$ & 0 \\
Elbow & $\{3\}$ & 0 & $l_{h}=0.055 m$ & $q_{3}$ & 0 \\
Wrist+digits & $\{4,5,6\}$ & 0 & $l_{r}=0.070 m$ & $q_{4,5,6}$ & 0 \\
\hline
\end{tabular}

about $z_{i}$.

The shoulder joint of the robot is composed by two degrees of freedom: $q_{1}, q_{2}$. The former angle allows for the primary flapping motion (rotation about axis $z_{1}$ ) whereas the latter allows the wings to rotate about the axis $z_{2}$. The elbow joint has one degree of freedom: $q_{3}$, which allows the wings to contract or extend in sync with the flapping motion. The wrist joint has three degrees of freedom: $q_{4}, q_{5}, q_{6}$. Each angle allows for the rotation of metacarpophalangeal (MCP) digits III, IV and V about axes $z_{4}, z_{5}, z_{6}$ respectively. Both elbow and wrist joints provide the morphing-wing capability to the robot.

\section{Body-frame}

The body frame $\{b\}$ has $x_{b}$ pointing cranially along the body axis, $y_{b}$ pointing laterally toward the right wing, and $z_{b}$ points downward and lies in the plane of symmetry of the body. The rotation of $\{b\}$ with respect to the fixed inertial frame $\{o\}$ is represented by the Euler angles: roll $(\phi)$, pitch $(\theta)$, and yaw $(\psi)$ following aerodynamic conventions [26]. In the inertial frame $\{o\}, x_{o}$ and $y_{o}$ describe the horizontal plane and $+z_{o}$ points in the direction of gravity.

\section{Rotations and translations}

Kinematics transformations that relate two consecutive frames of the wing are given by a $4 \times 4$ homogeneous transformation matrix $\left(T_{i+1, i}\right)$.

$$
T_{i+1, i}=\left[\begin{array}{cc}
r_{i+1, i} & p_{i, i+1} \\
0 & 1
\end{array}\right]=\left[\begin{array}{cccc}
\cos q_{i} & -\cos \alpha_{i} \sin q_{i} & \sin \alpha_{i} \sin q_{i} & a_{i} \cos q_{i} \\
\sin q_{i} & \cos \alpha_{i} \cos q_{i} & -\sin \alpha_{i} \cos q_{i} & a_{i} \sin q_{i} \\
0 & \sin \alpha_{i} & \cos \alpha_{i} & d_{i} \\
0 & 0 & 0 & 1
\end{array}\right]
$$

In Eq.(1) $r_{i+1, i}$ is the basic rotation matrix and $p_{i, i+1}$ the position vector. The terms $\alpha_{i}$, $a_{i}, d_{i}, q_{i}$, correspond to the geometrical DH parameters of the body $i$. Table 2 details these parameters.

\subsection{Dynamics}

Equations of Motion (EoM) are formulated based on the Newton-Euler formalism [23]. This leads to six-dimensional physical quantities that combine the angular and linear aspects of 


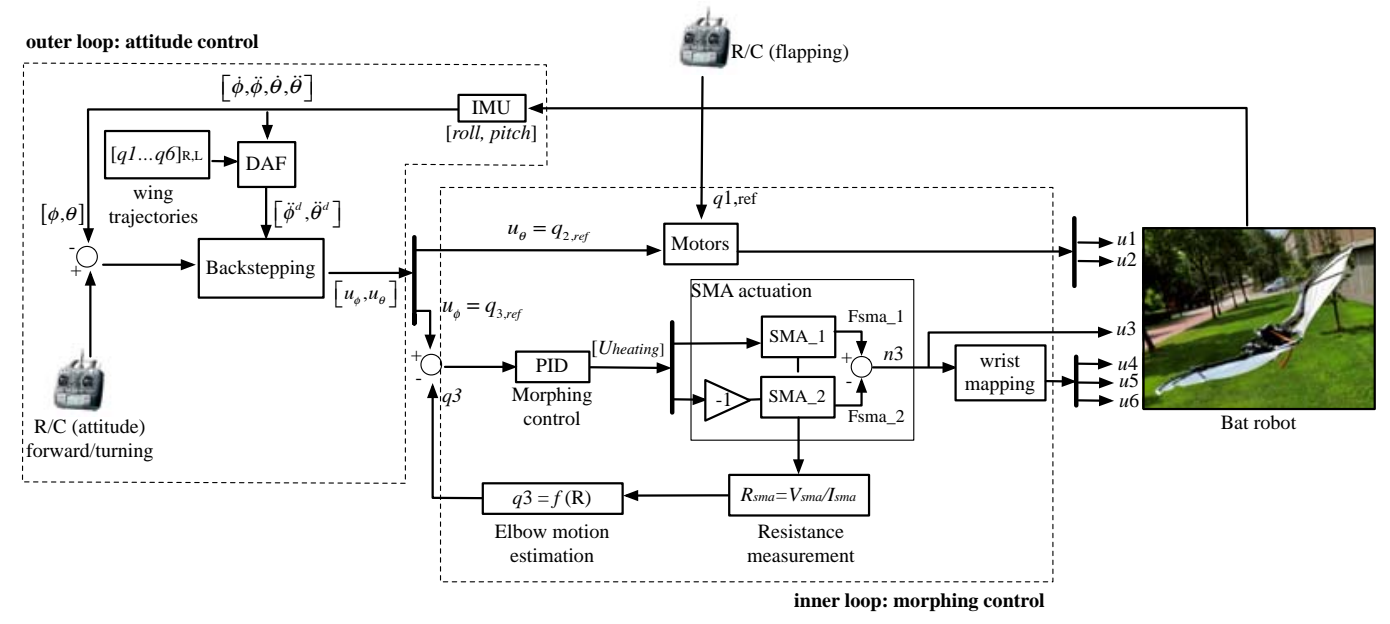

Figure 3. Flight control architecture scheme for one wing.

rigid-body motions and forces. Using spatial algebra notation, velocities $V_{i}$, accelerations $\dot{V}_{i}$, and forces $F_{i}$ are expressed with respect to joint frame $\{i\}$ using six-dimensional vectors, as:

$$
V_{i}=\left[\begin{array}{c}
\omega_{i} \\
v_{i}
\end{array}\right], \dot{V}_{i}=\left[\begin{array}{c}
\dot{\omega}_{i} \\
\dot{v}_{i}
\end{array}\right], F_{i}=\left[\begin{array}{c}
n_{i} \\
f_{i}
\end{array}\right] \in \mathfrak{R}^{6 \times 1}
$$

The inset in figure 2 details how the physical components in Eq. (2) are expressed in a rigid body $i$. Spatial forces acting on the center of mass of the robot $\left(F_{T}\right)$ are computed as determined by Eq. (3). It shows the sum of propagated forces produced by each wing, i.e., right $\left(F_{i, R}\right)$ and left $\left(F_{i, L}\right)$ respectively.

$$
F_{T}=\sum_{i=6}^{0} F_{i, R}+\sum_{i=6}^{0} F_{i, L} \in \mathfrak{R}^{6 x 1}
$$

Because $F_{T}$ is with respect to the base frame $\{0\}$, the rolling torque $\left(\tau_{\phi}\right)$ and pitching torque $\left(\tau_{\theta}\right)$ defined in figure 2 are extracted from vector $F_{T}$ and expressed with respect to body-frame $\{b\}$, as:

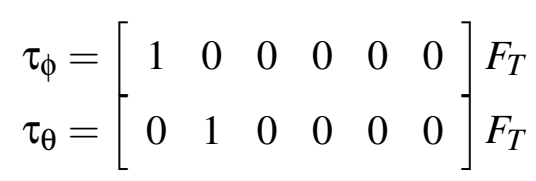

Torque around the $z_{b}$ axis (yaw) is not defined since the robot cannot directly control yaw motion. Section 3 details the procedure to compute $F_{T}$. In addition, simulation results are compared against experimental quantification of $F_{T}$. This allows validation of Eq. (3) and determination of the influence of wing inertia on the robot's maneuverability.

\subsection{Flight control}

Figure 3 shows the flight control architecture. It is composed of two control layers: i) attitude controller and ii) morphing-wing controller. The former consists on an outer closed-loop 
control that drives the inner one. Its purpose is to allow the robot to fly forward or turn by means of regulating the attitude variables roll $(\phi)$ and pitch $(\theta)$. The latter consists on an inner closed-loop control for extending and contracting both wings by means of elbow and wrist joint rotations $\left(q_{3} \ldots q_{6}\right)$.

\section{Control inputs}

In terms of actuation, a servo motor drives the flapping $\left(q_{1}\right)$ and pitch $\left(q_{2}\right)$ motions of the wings, whereas SMAs drive the morphing motion of the wings $\left(q_{3}\right)$. The main reason for this choice concerns the speed limitation of SMAs for achieving higher flapping frequencies.

- The primary flapping motion $q_{1}$ is directly mapped to the motor drive. It generates a control input $u_{1}$ that actuates the shoulder joints of both wings. This allows the robot to flap at the desired frequency $f$. The maximum wingbeat frequency is $8 \mathrm{~Hz}$.

- Pitch motion $(\theta)$ is achieved by modulating the joint angles $q_{2}$. Pitch is regulated by the backstepping+DAF controller which uses the IMU information to generate the reference $u_{\theta}$. This signal is directly mapped to the motor drive in order to generate the control input $u_{2}$, which actuates the shoulder joint $q_{2}$.

- Roll motion $(\phi)$ is achieved by modulating the elbow joint $q_{3}$. Roll is regulated by the backstepping+DAF controller which uses the IMU information to generate the reference $u_{\phi}$. This reference drives the inner loop. This inner loop consists of a PID controller that regulates the amount of input heating power $\left(U_{\text {heating }}\right)$ to be delivered to the antagonistic pair of SMA actuators. The antagonistic mechanism generates an output torque $\left(n_{3}\right)$ that directly actuates the elbow joint $\left(u_{3}=n_{3}\right)$. On the other hand, the wrist is composed by under-actuated joints $\left(q_{4} \ldots q_{6}\right)$ that rotate as a function of the elbow joint. To connect both joints, steel tendons have been placed inside the radius bone. These tendons allow for the motion of the metacarpophalangeal (MCP) digits III, IV and V which are attached to the wrist joint (cf. figure 1). Each digit joint has different radii, allowing for different rotation ranges. Using this approach, the digits open and close to maintain the proper tension of the wing membrane during the morphing-wing modulation. For simulation, the module wrist mapping in figure 3 determines the control inputs $u_{4} \ldots u_{6}$ as a function of $n_{3}$.

\section{Attitude regulation}

The attitude controller allows the bat-robot to maneuver by means of changing the wing shape, using the morphing-wing control mechanism presented in [19]. The attitude control goal is twofold:

(i) To regulate roll $(\phi)$ and pitch $(\theta)$ motions that allow the robot to properly maneuver.

(ii) To take advantage of wing inertia information to increment net forces $F_{\text {net }}$. 


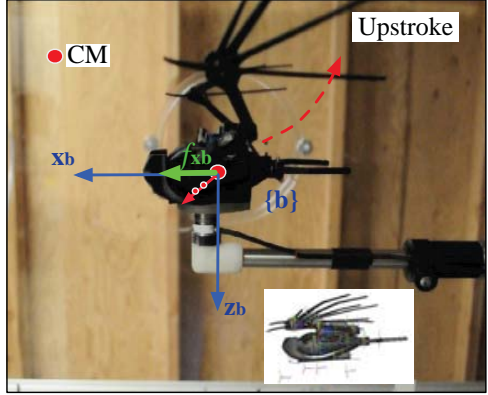

(a)

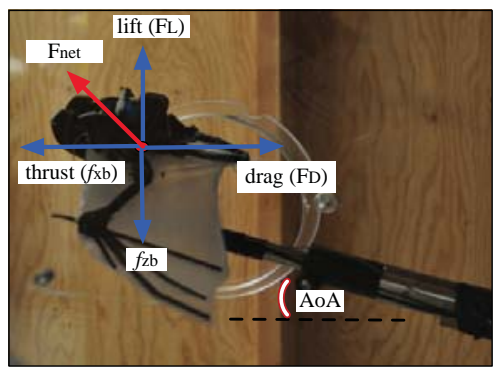

(c)

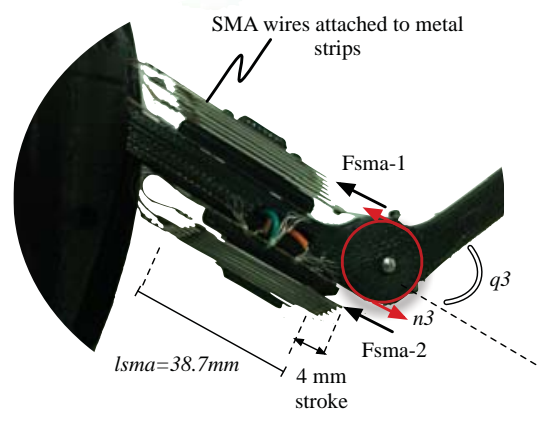

(e)

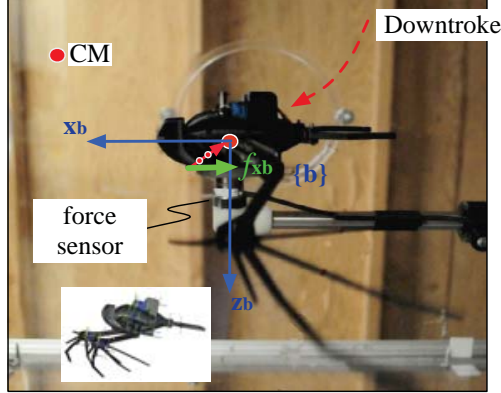

(b)

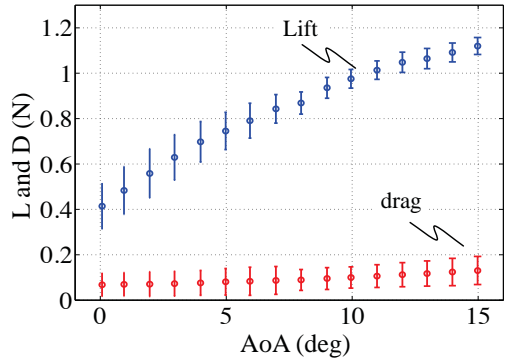

(d)

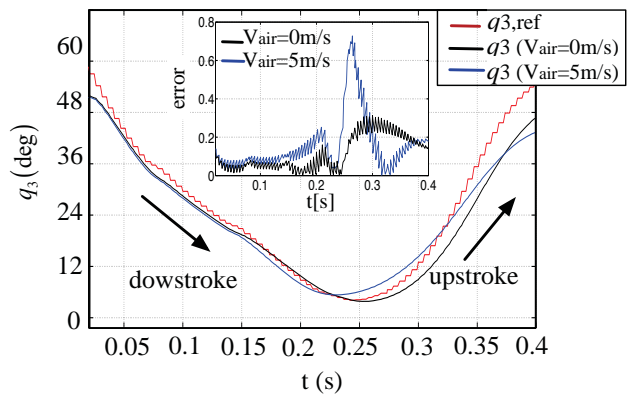

(f)

Figure 4. a-b) Inertial effects of the flapping motion of the wings on the position of the center of mass and accelerations of the body: (a) end of the wing upstroke, (b) end of the wing downstroke. c) bat-robot mounted on top of a 6-DoF force sensor from which both lift $F_{L}$ and drag $F_{D}$ forces are experimentally calculated as a function of the airflow speed and angle of attack, d) experimental quantification of lift and drag versus angle of attack (AoA), airspeed of $5 \mathrm{~ms}^{-1}$, e) antagonistic configuration of SMA actuators acting as artificial bicep and tricep muscles. The rotation of the elbow joint $q_{3}$ allows for the contraction and extension of the wing (morphing), f) close-up to a wingbeat cycle at $f=2.5 \mathrm{~Hz}$. The PID morphing controller allows the robot to track bio-inspired elbow joint references $q_{3, \text { ref }}$. The inset shows tracking errors produced at airspeeds $\left(V_{\text {air }}\right)$ of 0 and $5 m s^{-1}$.

The backstepping+DAF controller is aimed at achieving both goals. Backstepping has been widely applied to robust flight control problems [30]. The key idea of the backstepping design is to benefit from the desired dynamic state feedback that composes the control law $u=f\left(z, z^{d}\right)[31]$. In this case, $z$ corresponds to $(\ddot{\phi})$ or $(\ddot{\theta})$ respectively. The backstepping does 
not only ensure global asymptotically stabilisation of $\phi$ and $\theta$, it also allows for the definition of a Desired angular Acceleration Function (DAF) to define the terms $\left(\ddot{\phi}^{d}\right)$ and $\left(\ddot{\theta}^{d}\right)$.

The DAF terms are used to include wing inertia information that is required for the proper modulation of the wing joints. In bats, positive inertial thrust is produced during the upstroke motion of the wings mainly because the body moves in opposition to the flapping direction in order to conserve momentum. Bats properly modulate wing kinematics to take maximum advantage of wing inertia on the production of body accelerations. These accelerations are produced by the net forces $\left(F_{n e t}\right)$. As shown in figure $4 \mathrm{c}, F_{\text {net }}$ is calculated with respect to the body frame $\{b\}$, as:

$$
F_{n e t}=\left(\left[\begin{array}{llllll}
0 & 0 & 0 & 1 & 0 & 0
\end{array}\right] F_{T}-F_{D}\right)+\left(F_{L}-\left[\begin{array}{llllll}
0 & 0 & 0 & 0 & 0 & 1
\end{array}\right] F_{T}\right),
$$

where $F_{T} \in \mathfrak{R}^{6 x 1}$ is the sum of propagated inertial forces produced by each wing and the term $\left[\begin{array}{cccccc}0 & 0 & 0 & 1 & 0 & 0\end{array}\right] F_{T}$ is the linear force component $\left(f_{x b}\right)$ along the $x_{b}$ axis of frame $\{b\}$. In simulation, $F_{T}$ is computed using Eq. (3). In the experiment, $F_{T}$ is directly measured from the 6D force sensor located below the body (cf. Figure 4b). On the other hand, terms $F_{D}, F_{L}$ are the drag and lift forces experimentally measured in the wind-tunnel. Figure $4 \mathrm{~d}$ shows the aerodynamics data.

The net force produced $\left(F_{n e t}\right)$ can be decomposed into net force components parallel and perpendicular to the direction of flight. Net horizontal force is due to thrust-drag, whereas net vertical force is due to lift-weight. Also $F_{n e t}$ varies during the wingbeat cycle in part due to the inertial thrust (forward-oriented component, $f_{x b}$ ) as it changes from upstroke to downstroke and vice versa [13]. Figures $4 \mathrm{a}-\mathrm{b}$ explain this issue: to conserve momentum, the body moves in opposition to the flapping direction. During upstroke, the upward and backward acceleration caused by the flapping motions of the wings produce an inertial force (red circled arrow) that moves the body forward and downward with respect to the downstroke. This force produces a forward-oriented component $\left(f_{x b}\right)$ or inertial thrust (green solid arrow). Contrary, during the downstroke negative inertial thrust is produced.

\section{Morphing-wing regulation}

To change wing morphology, Shape Memory Alloys (SMAs) have been used as artificial muscles. The very light structure of the SMA actuators $(1.1 \mathrm{~g}$ each) make them suitable for the construction of light wings with muscle-like actuation similar to the one of biological bats. Figure 4e details the antagonistic pair of SMA actuators acting as biceps and triceps. The SMA actuators (supplied by Migamotors www . mi gamotors . com) consists of several short strips of Nitinol SMA wire attached to opposite ends of six metal strips stacked in parallel. Each SMA segment pulls the next strip about $0.67 \mathrm{~mm}$ relative to the previous strip, and the relative movements sum to make a stroke of $4 \mathrm{~mm}$. The elbow torque $n_{3}$ is generated by the difference between the pull forces $\left(F_{\text {sma }}\right)$ generated by each SMA actuator. The configuration shown in figure $4 \mathrm{e}$ allows an elbow rotation range of $\sim 60^{\circ}$. Previous work in [19] quantified the performance of SMA actuators for the application at hand. In summary, wing modulation is shown in figure 4f. During the downstroke, both wings extend via elbow joint rotation 
Table 3. Spatial operators

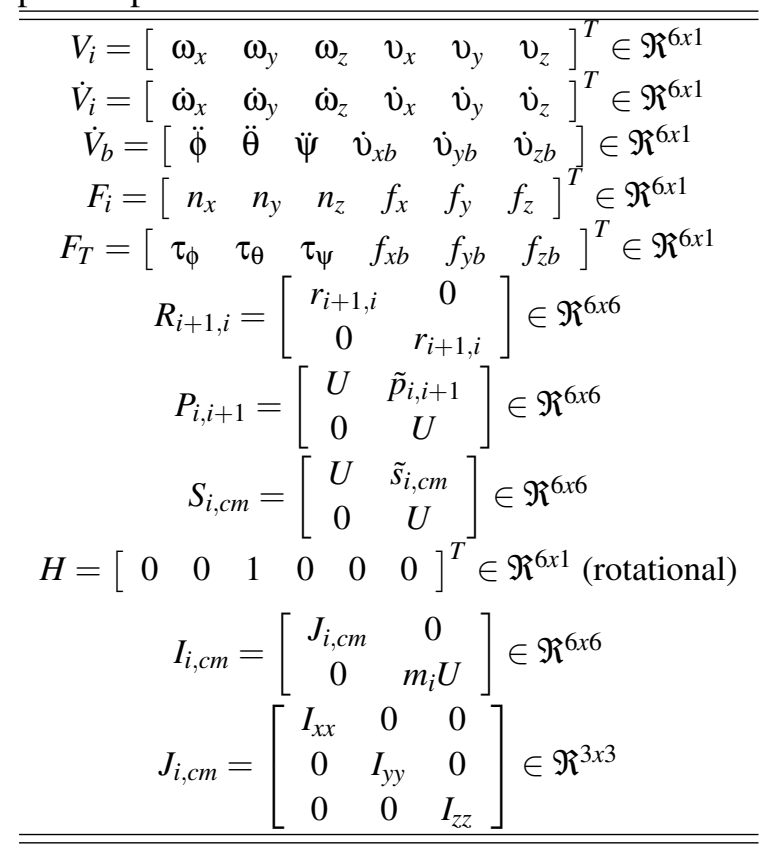

$\left(q_{3}\right)$ from $\sim 50^{\circ}$ to $\sim 5^{\circ}$, whereas during the upstroke, both wings retract again. Disparity in position tracking (see inset) is caused by accumulative errors introduced during the estimation of $q_{3}$ via SMA electrical resistance measurements (cf. [19]). Errors tend to be higher during the upstroke due to high aerodynamic loads caused by drag forces. This introduces serious disturbances that are difficult to completely reject, specially if the angular motion generated by $q_{3}$ is not directly sensed.

\section{The role of wing inertia for maneuvering}

To compute $F_{T}$ in Eq. (3) the spatial propagation of wing forces $\left(F_{i, R}, F_{i, L}\right)$ must be calculated. The following procedure describes how to recursively solve $F_{i}$ for the multi-body wing scheme shown in figure 2. The joint trajectory of each wing is composed by the joint positions $\left(q_{i}\right)$, velocities $\left(\dot{q}_{i}\right)$, and accelerations $\left(\ddot{q}_{i}\right)$.

\subsection{Equations of motion (EoM)}

Spatial operators from Table 3 are used to formulate the dynamics EoM. Spatial forces and velocities calculated with respect to the center of mass frame $\{\mathrm{cm}\}$ of a rigid body $i$ are related with respect to the joint frame $\{i\}$ of the body, as:

$$
\begin{aligned}
F_{i} & =S_{i, c m}^{T} F_{i, c m}, \\
V_{i} & =S_{i, c m}^{T} V_{i, c m}
\end{aligned}
$$

By definition, the spatial force acting on the center of mass of a body $i$ can be expressed by differentiating the spatial inertial moment $\left(L_{i, c m}=I_{i, c m} V_{i, c m}\right)$ with respect to time, as:

$$
F_{i, c m}=\dot{L}_{i, c m}=I_{i, c m} \dot{V}_{i, c m}+\dot{I}_{i, c m} V_{i, c m}
$$


Substituting Eq. (7) into Eq. (6) yields:

$$
F_{i}=S_{i, c m}^{T}\left[I_{i, c m} \dot{V}_{i, c m}+\xi\right]
$$

where $\xi=\dot{I}_{i, c m} V_{i, c m}$ is the gyroscopic force acting on the center of mass frame $\{\mathrm{cm}\}$ of the rigid body $i$. The spatial acceleration $\dot{V}_{i, c m}$ can be solved by differentiating $V_{i, c m}$ in Eq. (6) with respect to time, as:

$$
\begin{aligned}
V_{i, c m} & =S_{i, c m} V_{i}, \\
\dot{V}_{i, c m} & =S_{i, c m} \dot{V}_{i}+\dot{S}_{i, c m} V_{i}
\end{aligned}
$$

Substituting $\dot{V}_{i, c m}$ in Eq.(9) into $F_{i}$ in Eq.(8) gives:

$$
F_{i}=S_{i, c m}^{T}\left[I_{i, c m}\left(S_{i, c m} \dot{V}_{i}+\dot{S}_{i, c m} V_{i}\right)+\xi\right] .
$$

The term $\dot{S}_{i, c m} V_{i}$ contains Coriolis effects. The spatial forces in Eq. (10) can be recursively propagated along the wing by considering the spatial operators for rotation $R_{i+1, i}$ and translation $P_{i, i+1}$. These spatial operators allow for the projection and rotation of the EoM relating two consecutive frames (based on homogeneous transformations, cf. Eq. (1)). Therefore, spatial forces in Eq. (10) are backward propagated, from the wingtip $(i=6)$, to the base frame $\{0\}(i=0)$, as:

$$
\begin{aligned}
& \sum_{i=6}^{0} F_{i}=R_{i+1, i} P_{i, i+1}^{T} F_{i+1}+S_{i, c m}^{T}\left[I_{i, c m}\left(S_{i, c m} \dot{V}_{i}+\dot{S}_{i, c m} V_{i}\right)+\xi\right] \\
& =R_{i+1, i} P_{i, i+1}^{T} F_{i+1}+S_{i, c m}^{T} I_{i, c m} S_{i, c m} \dot{V}_{i}+S_{i, c m}^{T} I_{i, c m} \dot{S}_{i, c m} V_{i}+\dot{I}_{i, c m} S_{i, c m}^{T} V_{i, c m}
\end{aligned}
$$

At the beginning of the propagation $(i=6)$, the term $F_{i+1}=0$ in the absence of external forces. When aerodynamic forces are applied to the model, the term $F_{i+1}$ (only when $i=6$ ) would correspond to: $F_{i+1}=\left[\begin{array}{cccccc}0 & 0 & 0 & -F_{D} & 0 & F_{L}\end{array}\right]^{T}$. For the rest of the propagation, the expression $R_{i+1, i} P_{i, i+1}^{T} F_{i+1}$ allows for the projection of spatial forces along the serial chain of bodies that compose the wing structure. Also note from Eq. (11) that the term $S_{i, c m}^{T} I_{i, c m} S_{i, c m}$ refers to the spatial inertia $I_{i}$ calculated with respect to the joint frame $\{i\}$ by applying the parallel axis theorem. Equation (11) is rewritten by substituting the terms $S_{i, c m}^{T} I_{i, c m} S_{i, c m}$ and $S_{i, c m}^{T} I_{i, c m}$ by $I_{i}$, and $S_{i, c m}^{T} V_{i, c m}$ by $V_{i}$ :

$$
\sum_{i=6}^{0} F_{i}=R_{i+1, i} P_{i, i+1}^{T} F_{i+1}+I_{i} \dot{V}_{i}+\left[I_{i} \dot{S}_{i, c m}+\dot{I}_{i}\right] V_{i} .
$$

To complete the solution of the inertial model in Eq. (12), the set of spatial velocities $\left(V_{i}\right)$ and accelerations $\left(\dot{V}_{i}\right)$ are also recursively calculated as:

$$
\begin{aligned}
& V_{i}=P_{i, i+1} R_{i+1, i}^{T} V_{i-1}+H \dot{q}_{i} \\
& \dot{V}_{i}=P_{i, i+1} R_{i+1, i}^{T} \dot{V}_{i-1}+\dot{P}_{i, i+1} \dot{R}_{i+1, i}^{T} V_{i-1}+H \ddot{q}_{i}+\dot{H} \dot{q}_{i}
\end{aligned}
$$




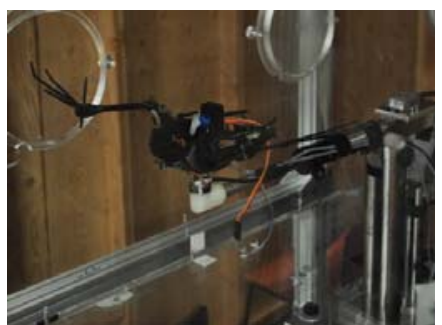

(a)

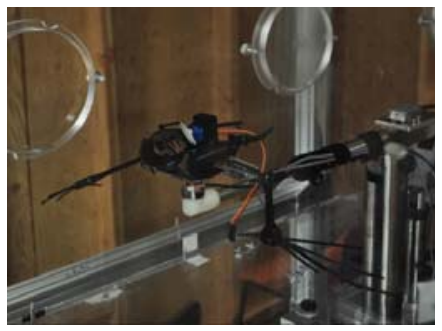

(c)

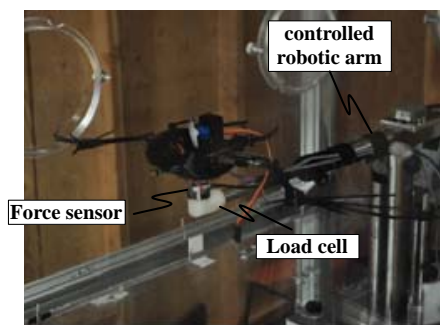

(b)

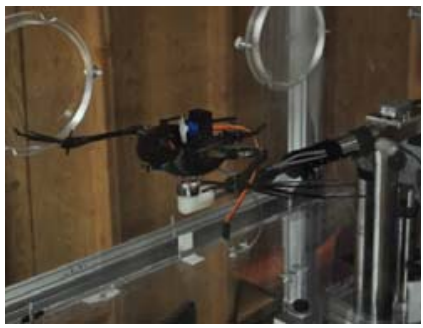

(d)

Figure 5. Inertial force measurements: a) beginning of downstroke, b) middle downstroke (wings extended), c) beginning of upstroke, d) middle upstroke (wings retracted). The wing-membrane has been removed to quantify inertial effects.

\subsection{Maneuvers}

Having the inertial model completely formulated in Eqs. (12) and (13), the force contribution produced by the wings can be modelled by solving $F_{T}$ in Eq. (3). To validate the accuracy of the inertial model, experimental measurements of $F_{T}$ have been carried out. Figure 5 describes the testbed, which shows the bat-robot inside the wind tunnel, on the end of a supporting sting that defines the angle of attack. The robot is mounted on top of a 6-DoF force sensor from which $F_{T}$ has been experimentally quantified. The force sensor corresponds to a Nano17 transducer ATI Industrial Automation with 0.318 gram-force of resolution. The setup is aimed at quantifying inertial measurements. Possible aerodynamic loads caused by the wing skeleton are not relevant compared to the inertial contribution.

To successfully complete a turn, bats must translate their center of mass along the flight path (i.e. change its flight direction) and rotate their body around their center of mass to align its body orientation with the new direction. The net forces generated by the body and wings produce the change of orientation, allowing the animal to turn. Based on this foundation, turning flight can be achieved by generating roll momentum by means of wing contraction and extension. Figure 6 shows the wing modulation scheme that allows for the displacement of the center of mass of the robot towards the expanded wing. This approach allows the robot to roll $(\phi)$.

The set of joint trajectories $q_{i}, \dot{q}_{i}, \ddot{q}_{i}$ that allow the wing modulation scheme described in figure 6 is completely detailed in figure 7 . The joint trajectory patterns have been extracted from the bio-inspired analysis of bat turning flight carried out in [12], [24] and [32].

To generate forward pitching torque $\left(\tau_{\theta}\right)$ both wings must be positioned towards the body (see inset from figure $8 \mathrm{a}$ ). The blue plot corresponds to the experimental values of $\tau_{\theta}$ 


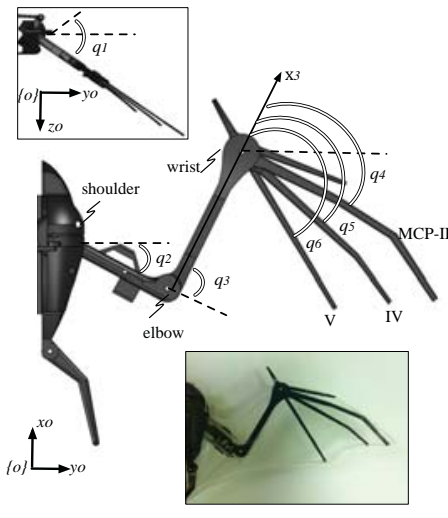

(a)

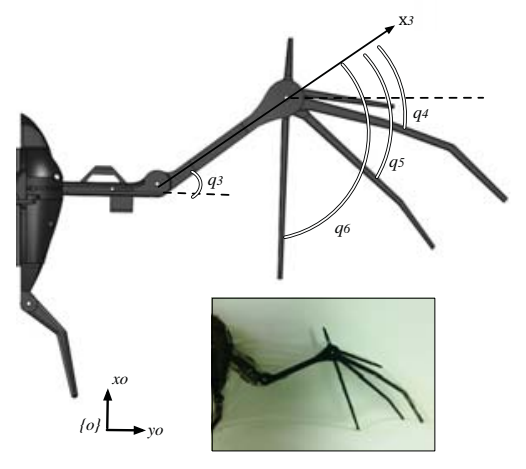

(b)

Figure 6. Wing modulation scheme for turning flight $(\phi)$ : a) wing contraction during upstroke, b) wing extension during downstroke.
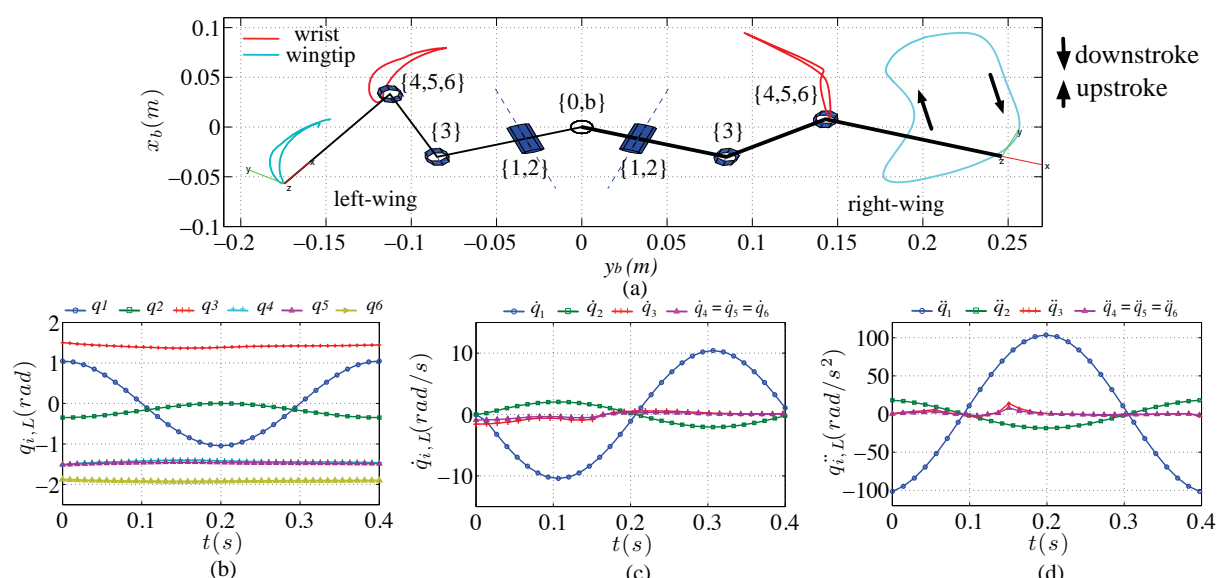

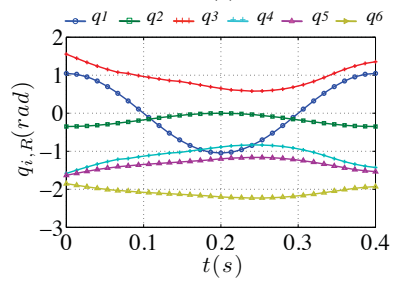

(e)

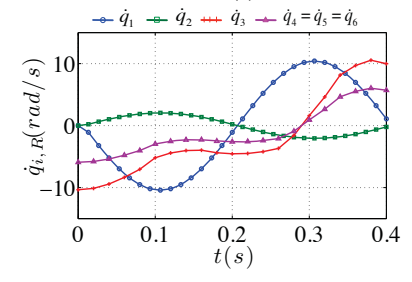

(f)

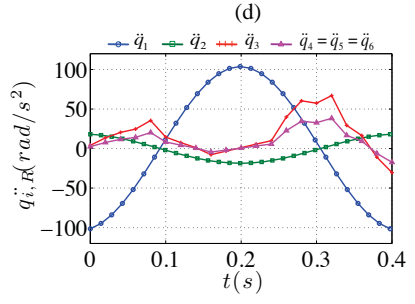

(g)

Figure 7. Kinematics of turning flight: a) Cartesian trajectories for the wrist and wingtip frames during a wingbeat cycle, b)-c)-d) joint trajectory profile for the contracted left wing $(f=2.5 \mathrm{~Hz}), \mathrm{e})-\mathrm{f})-\mathrm{g})$ joint trajectory profile for the extended right wing $(f=2.5 H z)$.

measured by the force sensor, whereas the black plot corresponds to the simulated response of $\tau_{\theta}$ computed in Eq. (4). The simulated pitching torque has been extracted from $F_{T}$ as defined in Eq. (4). The disparity in results between the simulation model and the experimental results are due to two factors. Firstly, mechanical asymmetries due to small fabrication errors have caused the robot to generate a small component of pitching torque $\left(\tau_{\theta}=\sim 0.06 \mathrm{Nm}\right.$ ) even when $q_{2}=0^{o}$ (from $t=0 s$ to $t=2 s$ in figure $8 \mathrm{a}$ ). The solution of this problem requires 

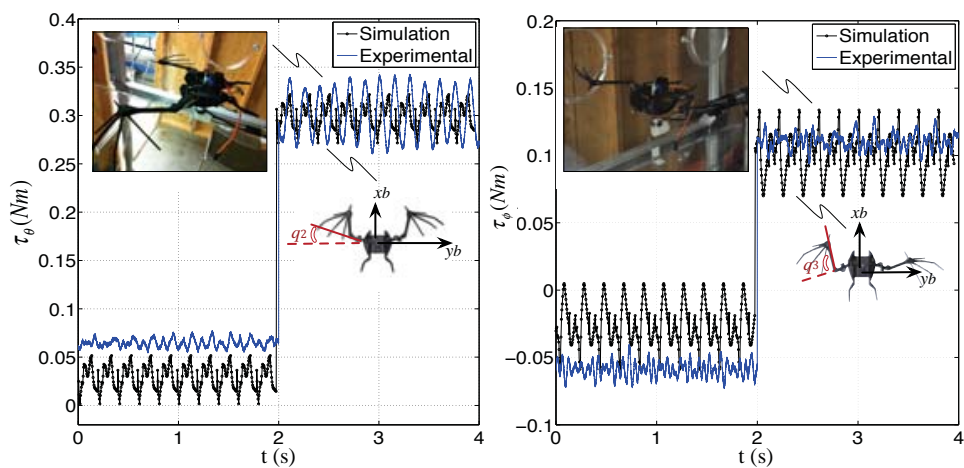

(a)
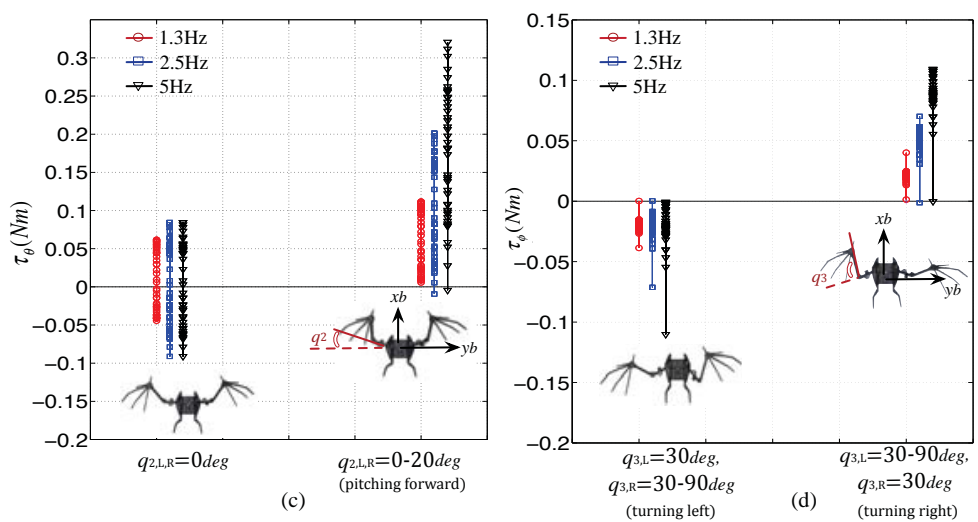

Figure 8. Wing inertia contribution: a) simulation model VS experimental results of pitching torques $\tau_{\theta}(f=5 \mathrm{~Hz})$, b) simulation model VS experimental results of rolling torques $\left.\tau_{\phi}(f=5 \mathrm{~Hz}), \mathrm{c}\right)$-d) quantification of wing inertia contribution into the generation of $\tau_{\theta}$ and $\tau_{\phi}$ at different wingbeat frequencies $f$.

the incorporation of an attitude controller that regulates the pitch motion. Secondly, the disparity in the amplitude of the oscillations is due to non-modelled physical quantities, such as mechanical friction. This issue may cause the simulation values to be larger in amplitude in comparison to the experimental readings.

To generate rolling torque $\left(\tau_{\phi}\right)$ that allows the robot to turn left or right one wing must be contracted while the other is extended (see inset from figure $8 \mathrm{~b}$ ). In this experiment, similar disparity problems to those observed from the pitch test scenario are seen. However, note from both figure 8a-b that from $t=2 s$ to $t=4 s$ the model and the experimental values tend to stabilise about the same bias value of torque. In figure 8 a the bias value of $\tau_{\theta}=\sim 0.31 \mathrm{Nm}$, whereas in figure $8 \mathrm{~b}$ the bias value of $\tau_{\phi}=\sim 0.11 \mathrm{Nm}$. Figure $8 \mathrm{c}$-d shows how bias values of $\tau_{\theta}$ and $\tau_{\phi}$ scale up when the wingbeat frequency increases. Table 4 details the numerical results of the scaling factors for both pitching and rolling torques.

It has been observed that the influence of wing and body mass $\left(M_{b}=0.125 \mathrm{Kg}\right)$ on the production of inertial torques is proportional to $M_{b}^{1 / f}, \forall f>0$. Biological studies in [13], [14], [32] show experimental evidence about the implication of this relationship in terms of inertial and aerodynamics contribution. For the application at hand, $M_{b}$ has accounted for $\sim 50 \%$ of pitching torque production and $\sim 20 \%$ of rolling torque production. 
Table 4. Wing and body mass influence in the generation of $\tau_{\theta}$ and $\tau_{\phi}\left(M_{b}=0.125 \mathrm{~kg}\right)$

\begin{tabular}{ccccc}
\hline \hline Scaling factor & & $f=1.3 \mathrm{~Hz}$ & $f=2.5 \mathrm{~Hz}$ & $f=5 \mathrm{~Hz}$ \\
\hline \multirow{2}{*}{$\operatorname{Pitch}^{1}\left(0.5 \mathrm{M}_{b}^{1 / f}\right)$} & Experimental & $0.11 \mathrm{Nm}$ & $0.2 \mathrm{Nm}$ & $0.31 \mathrm{Nm}$ \\
& Simulation & $0.1 \mathrm{Nm}$ & $0.21 \mathrm{Nm}$ & $0.33 \mathrm{Nm}$ \\
\hline \multirow{2}{*}{$\operatorname{Roll}^{2}\left(0.2 \mathrm{M}_{b}^{1 / f}\right)$} & Experimental & $0.04 \mathrm{Nm}$ & $0.07 \mathrm{Nm}$ & $0.11 \mathrm{Nm}$ \\
& Simulation & $0.04 \mathrm{Nm}$ & $0.08 \mathrm{Nm}$ & $0.13 \mathrm{Nm}$ \\
\hline \hline
\end{tabular}

1 Values from figure 8c

2 Values from figure $8 \mathrm{~d}$

\section{Attitude control}

This section addresses the attitude control problem by formulating the backstepping+DAF controller. The flight control architecture is shown in figure 3.

The robot is equipped with an Arduino-nano (http://arduino.cc) composed by a Atmega-328 microprocessor running at $16 \mathrm{MHz}$ and an Inertial Measure Unit (IMU) that includes 3-axis accelerometer and 3-axis gyroscope (http://www.sparkfun.com/ products /10121). The Backstepping+DAF controller uses the attitude data feedback by the IMU in order to generate the control commands $u_{\theta}$ and $u_{\phi}$ which drive the morphing-wing modulation.

The attitude data $(\theta, \dot{\theta}, \phi, \dot{\phi})$ and the reference trajectories of the wings $\left(q_{1} \ldots q_{6}\right)$ are required to formulate the Desired angular Acceleration Function (DAF). Desired roll and pitch angular accelerations are determined using the DAF.

\subsection{Desired angular Acceleration Function (DAF)}

To formulate the DAF terms $\left(\ddot{\phi}^{d}, \ddot{\theta}^{d}\right)$, the attitude data $(\theta, \dot{\theta}, \phi, \dot{\phi})$ that is feedback by the IMU and the wing joint trajectories of reference $\left(q_{i}\right)$ are required, as described in the Flight control architecture from figure 3. Therefore DAF terms can be written as a function of attitude and wing modulation data as:

$$
\begin{aligned}
\ddot{\phi}^{d} & =f(\phi, \dot{\phi}, \dot{q}, \ddot{q}) \\
\ddot{\theta}^{d} & =f(\theta, \dot{\theta}, \dot{q}, \ddot{q})
\end{aligned}
$$

The desired roll and pitch angular accelerations $(\ddot{\phi}, \ddot{\theta})$ are components of the sixdimensional body accelerations $\dot{V}_{b}$ that are produced by the inertial forces $F_{T}$. The definition of $\dot{V}_{b}$ with respect to the body frame $\{b\}$ requires the computation of the inertial model from Eqs. (12) and (13) and yields:

$$
\dot{V}_{b}=\left(I_{b}+\left[R_{0, b} \sum_{i=6}^{0}\left(R_{i+1, i} P_{i, i+1}^{T} I_{i, R}\right)+R_{0, b} \sum_{i=6}^{0}\left(R_{i+1, i} P_{i, i+1}^{T} I_{i, L}\right)\right]\right)^{-1} F_{T}
$$

In Eq. (15), $I_{b}$ is the spatial inertia of the robot's body calculated with respect to the body frame $\{b\}$, whereas the term $\sum_{i=6}^{0} I_{i}$ expresses the propagation of wing inertias of both 
wings onto the base frame $\{0\}$. Subscripts ${ }_{R}$ and ${ }_{L}$ refer to the right and left wing respectively. The term $R_{0, b}$ applies a rotation to express wing inertias with respect to the body frame $\{b\}$. Finally, DAF terms are defined and expressed with respect to the body frame $\{b\}$ as:

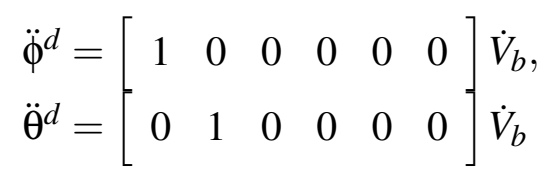

\subsection{Backstepping $+D A F$}

This section shows roll-control derivation for $u_{\phi}$. Pitch-control derivation $\left(u_{\theta}\right)$ follows the same procedure. The first step is to define the roll tracking error $e_{1}$ and its dynamics (derivative with respect to time):

$$
\begin{aligned}
& e_{1}=\phi^{d}-\phi \\
& \dot{e}_{1}=\dot{\phi}^{d}-\omega_{y}
\end{aligned}
$$

The term $\phi^{d}$ corresponds to the desired roll trajectory profile, whereas $\phi$ is the filtered roll angle measured by the IMU sensor. A positive definite Lyapunov function $(L)$ is used for stabilising the tracking error $e_{1}$, as:

$$
L\left(e_{1}\right)=\frac{e_{1}^{2}}{2},
$$

To regulate the behaviour of the angular velocity $\omega_{y}$ from $\dot{e}_{1}$ in Eq. (17), a second tracking error $e_{2}$ is defined, as:

$$
e_{2}=\omega_{y}^{d}-\omega_{y}
$$

The desired behaviour for $e_{2}$ is defined, as:

$$
\omega_{y}^{d}=c_{1} e_{1}+\dot{\phi}^{d}+\lambda_{1} \int e_{1},
$$

where $c_{1}, \lambda_{1}$ are positive constants and $\int e_{1}$ is the integral of the roll tracking error. In other words, $\omega_{y}^{d}$ is considered as a virtual control law that governs the behaviour of $e_{2}$. Substituting $\omega_{y}^{d}$ into Eq. (19) and differentiating $e_{2}$ with respect to time (note that $\omega_{y}=\dot{\phi}$ ):

$$
\dot{e}_{2}=c_{1} \dot{e}_{1}+\ddot{\phi}^{d}+\lambda_{1} e_{1}-\ddot{\phi}
$$

From Eq. (19), $\omega_{y}=\omega_{y}^{d}-e_{2}$. Substituting $\omega_{y}$ into $\dot{e}_{1}$ from Eq. (17) gives:

$$
\dot{e}_{1}=\dot{\phi}^{d}-\omega_{y}^{d}+e_{2}
$$

Substituting $\omega_{y}^{d}$ from Eq. (20) into (22):

$$
\begin{aligned}
& \dot{e}_{1}=\dot{\phi}^{d}-\left(c_{1} e_{1}+\dot{\phi}^{d}+\lambda_{1} \int e_{1}\right)+e_{2} \\
& \dot{e}_{1}=e_{2}-c_{1} e_{1}-\lambda_{1} \int e_{1}
\end{aligned}
$$

Finally, substituting $\dot{e}_{1}$ from Eq. (23) into Eq. (21):

$$
\dot{e}_{2}=c_{1}\left(e_{2}-c_{1} e_{1}-\lambda_{1} \int e_{1}\right)+\ddot{\phi}^{d}+\lambda_{1} e_{1}-\ddot{\phi}
$$


By expressing $\ddot{\phi}$ in terms of the pitching torque $\tau_{\phi}$ and the moment of inertia of the robot's body $I_{b, x x}$ about the $x_{b}$ axis of the body frame $\{b\}$, it is found that $\ddot{\phi}=\tau_{\phi} / I_{b, x x}$. Defining $u_{\phi}=\tau_{\phi}$, Eq. (24) can be written as:

$$
u_{\phi}=I_{b, x x}\left[c_{1}\left(e_{2}-c_{1} e_{1}-\lambda_{1} \int e_{1}\right)+\ddot{\phi}^{d}+\lambda_{1} e_{1}-\dot{e}_{2}\right]
$$

Substituting $\dot{e}_{2}=-e_{1}-\lambda_{2} e_{2}$ into Eq. (25), the backstepping+DAF control law in charge of roll regulation is derived in Eq. (26).

$$
u_{\phi}=I_{b, x x}\left[e_{1}\left(\lambda_{1}-c_{1}^{2}+1\right)+e_{2}\left(c_{1}+\lambda_{2}\right)-c_{1} \lambda_{1} \int e_{1}+\ddot{\phi}^{d}\right]
$$

The parameters of the controller, $\lambda_{1}, c_{1}, \lambda_{2}>0$ are defined in Table 5. By applying the same procedure, the backstepping+DAF control law in charge of pitch regulation is given by:

$$
u_{\theta}=I_{b, y y}\left[e_{3}\left(\lambda_{3}-c_{2}^{2}+1\right)+e_{4}\left(c_{2}+\lambda_{4}\right)-c_{2} \lambda_{3} \int e_{3}+\ddot{\theta}^{d}\right]
$$

By substituting the DAF terms $\ddot{\phi}^{d}, \ddot{\theta}^{d}$ from Eq. (16) into Eq. (26) and (27) respectively, gives:

$$
\begin{aligned}
& u_{\phi}=I_{b, x x}\left[e_{1}\left(\lambda_{1}-c_{1}^{2}+1\right)+e_{2}\left(c_{1}+\lambda_{2}\right)-c_{1} \lambda_{1} \int e_{1}+\left[\begin{array}{cccccc}
1 & 0 & 0 & 0 & 0 & 0
\end{array}\right] \dot{V}_{b},\right. \\
& u_{\theta}=I_{b, y y}\left[e_{3}\left(\lambda_{3}-c_{2}^{2}+1\right)+e_{4}\left(c_{2}+\lambda_{4}\right)-c_{2} \lambda_{3} \int e_{3}+\left[\begin{array}{llllll}
0 & 1 & 0 & 0 & 0 & 0
\end{array}\right] \dot{V}_{b}\right.
\end{aligned}
$$

Stability Analysis

The following candidate Lyapunov function has been chosen:

$$
L=0.5\left(e_{1}^{2}+e_{2}^{2}+\int e_{1}^{2}\right)
$$

Differentiating Eq. (29) with respect to time, having $\dot{e}_{1}=e_{2}-c_{1} e_{1}-\lambda_{1} \int e_{1}$ and $\dot{e}_{2}=-e_{1}-\lambda_{2} e_{2}$ gives,

$$
\begin{aligned}
& \dot{L}=e_{1} \dot{e}_{1}+e_{2} \dot{e}_{2}+e_{1}^{2} \leq 0 \\
& =e_{1}\left(e_{2}-c_{1} e_{1}-\lambda_{1} \int e_{1}\right)+e_{2}\left(-e_{1}-\lambda_{2} e_{2}\right)+e_{1}^{2} \\
& =-c_{1} e_{1}^{2}-\lambda_{2} e_{2}^{2} \leq 0
\end{aligned}
$$

The fact that Eq. (30) fulfils $\dot{L} \leq 0, \forall\left(e_{1}, e_{2}\right)$ ensures the boundedness of $e_{1}$, its integral $\int e_{1}$, and $e_{2}$. Hence, the reference angular value $\phi^{d}$ and the roll angle $\phi$ are also bounded due to $e_{1}=\phi^{d}-\phi$. The boundedness of $e_{1}$ implies that the virtual law $\omega_{y}^{d}$ is bounded as well, which consequently makes the error dynamics $\dot{e}_{2}$ and the DAF term $\ddot{\phi}^{d}$ also bounded. Furthermore, global asymptotic stability is also ensured due to the positive definition of $L$, in which $\dot{L}\left(e_{1}, e_{2}\right)<0, \forall\left(e_{1}, e_{2}\right) \neq 0$, and $\dot{L}(0)=0$ (by applying LaSalle's theorem). 
Table 5. List of robot's parameters: morphological, modelling, control.

\begin{tabular}{ccc}
\hline \hline Parameter (unit) & Robot & Specimen \\
\hline$M_{b}(\mathrm{~g})$ & 125 & 98 \\
$m_{w}[\mathrm{~g}]$ & 46.15 & 33.16 \\
$B(\mathrm{~m})$ & 0.245 & 0.462 \\
$l_{m}(\mathrm{~m})$ & 0.04 & 0.07 \\
{$\left[I_{b, x x}, I_{b, y y}, I_{b, z z}\right]\left(\mathrm{gcm}^{2}\right)$} & {$[1,0.07,0]$} & - \\
$S=l_{m}+2 B(\mathrm{~m})$ & 0.53 & 0.99 \\
$A_{b}\left(\mathrm{~m}^{2}\right)$ & 0.05 & 0.069 \\
$l_{h}(\mathrm{~m})$ & 0.055 & 0.11 \\
{$\left[I_{2, x x}, I_{2, y y}, I_{2, z z}\right]\left[\mathrm{cm}^{2}\right]$} & {$[0.03,0.37,0.93]$} & - \\
$s_{2, c m}(\mathrm{~m})$ & {$[0.0275,0,0]$} & - \\
$l_{r}(\mathrm{~m})$ & 0.070 & 0.145 \\
{$\left[I_{3, x x}, I_{3, y y}, I_{3, z z}\right]\left(g \mathrm{~cm}^{2}\right)$} & {$[0.07,0.92,0.37]$} & - \\
$s_{3, c m}(\mathrm{~m})$ & {$[0.035,0,0]$} & - \\
$\rho_{a i r}\left(K g / m^{3}\right)$ & 1.2 & - \\
$C_{L} 1$ & 1.5 & - \\
$C_{D} 1$ & 0.152 & - \\
$F_{L}{ }^{1}(\mathrm{~N})$ & 0.97 & - \\
$F_{D}{ }^{1}(\mathrm{~N})$ & 0.099 & - \\
{$\left[\lambda_{1}, c_{1}, \lambda_{2}\right]$} & {$[1.87,2.1,0.02]$} & - \\
{$\left[\lambda_{3}, c_{2}, \lambda_{4}\right]$} & {$[4.5,2.5,0.02]$} & - \\
\hline \hline
\end{tabular}

${ }^{1}$ Values experimentally measured at angle of attack of $9^{\circ}$, wind-tunnel airspeed of $V_{\text {air }}=5 \mathrm{~ms}^{-1}$ and wingbeat frequency of $f=2.5 \mathrm{~Hz}$ (cf. figure $4 \mathrm{~d}$ )

\section{Attitude control results}

This section presents wind-tunnel experimental results regarding the performance of the proposed attitude controller in terms of:

(i) evaluating the accuracy of the backstepping+DAF controller for tracking pitch and roll references under the presence of external disturbances caused by aerodynamic loads at airspeeds up to $5 m s^{-1}$,

(ii) demonstrating the assumption of incrementing net body forces $\left(F_{n e t}\right)$ thanks to the wing modulation driven by the backstepping+DAF controller.

Figure 9 describes the experimental setup. The bat robot is equipped with onboard processor based on Arduino technology which uses a PIC18F2680 that mainly receives the commands from the external PC (Matlab environment) via serial connection. These commands correspond to the control outputs for: flapping $u_{1}$ and wing modulation $u_{\phi}, u_{\theta}$ (subscripts $R$ and $L$ refer to the right and left wing respectively). The SMA driver is based on a MOSFET transistor that receives the control command $u_{3}$ and generates the driving electrical current signal to operate each SMA actuator of the wings. The robot is also equipped with onboard IMU that feedbacks attitude measurements: $\ddot{\phi}, \dot{\phi}, \ddot{\theta}, \dot{\theta}$. SMA electrical resistance is also measured and feedback $\left(R_{s m a}\right)$. A cell load within the wind-tunnel equipped with a force 


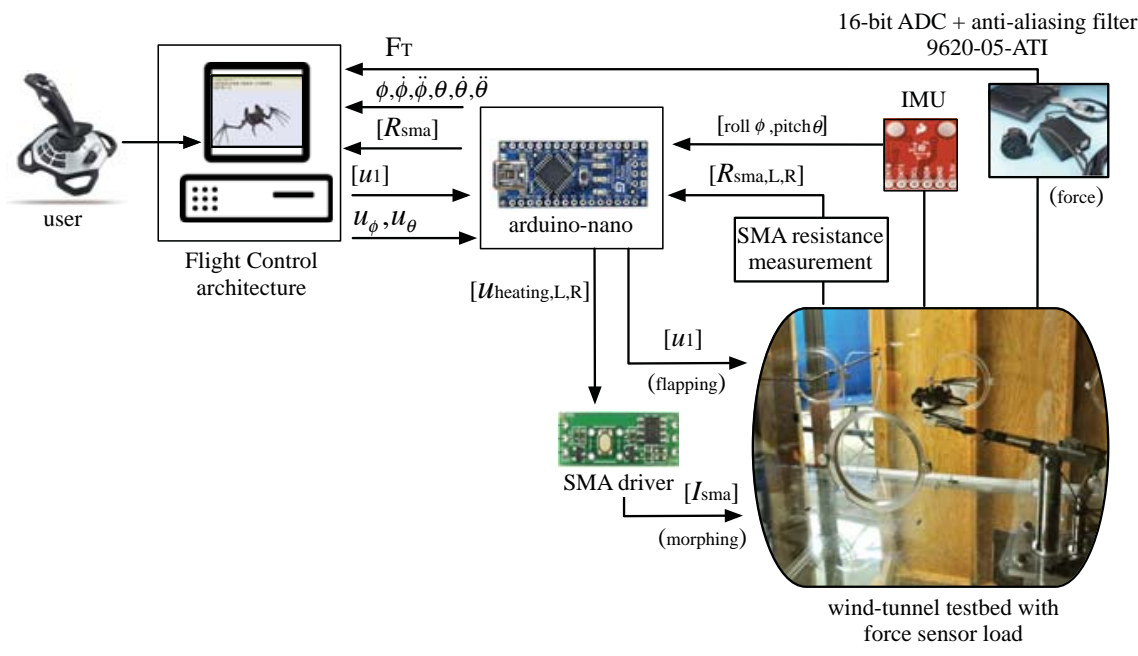

Figure 9. Experimental setup.
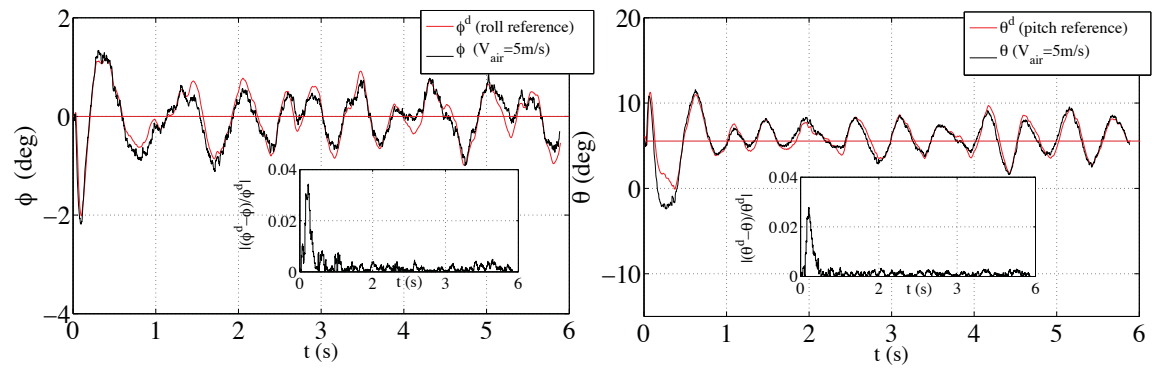

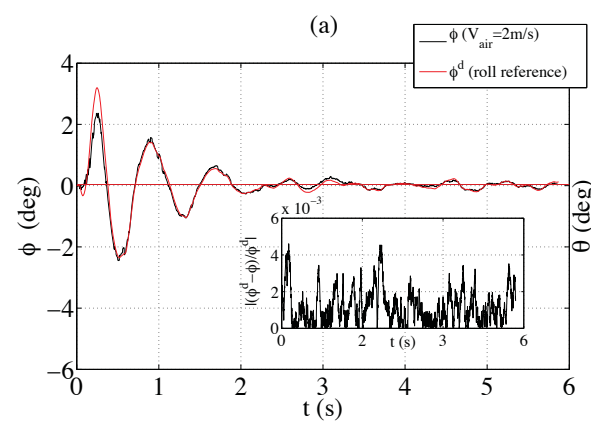

(c)

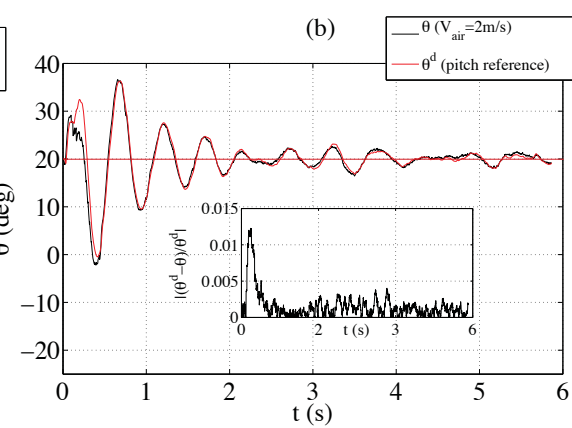

(d)

Figure 10. Forward flight control. Backstepping+DAF attitude tracking at: a)-b) roll and pitch tracking with $V_{\text {air }}=5 m s^{-1}$, c)-d) roll and pitch tracking with $V_{\text {air }}=2 m s^{-1}$.

sensor allows the quantification of 6D forces produced at the center of mass of the robot $\left(F_{T}\right)$. A 16-bit DAC with embedded $1^{\text {st }}$ order anti-aliasing filter is used for that purpose. Attitude outer loop based on IMU readings runs at $20 \mathrm{~Hz}$, whereas morphing inner loop based on SMA resistance readings runs at $30 \mathrm{~Hz}$. Details on these control loops are depicted in figure 3. Table 5 describes the numerical values for both modelling and control parameters. 


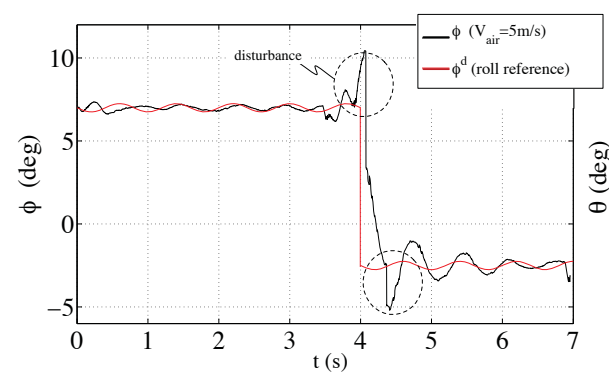

(a)

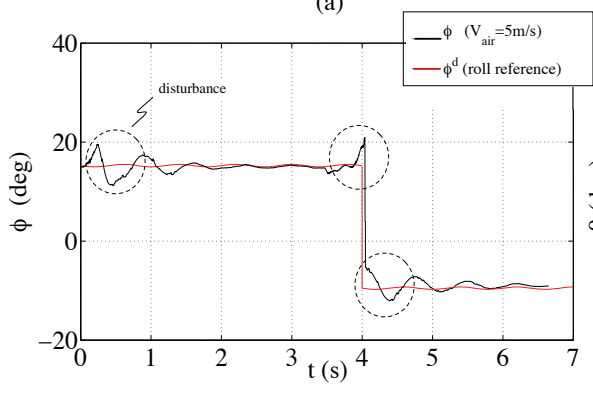

(c)

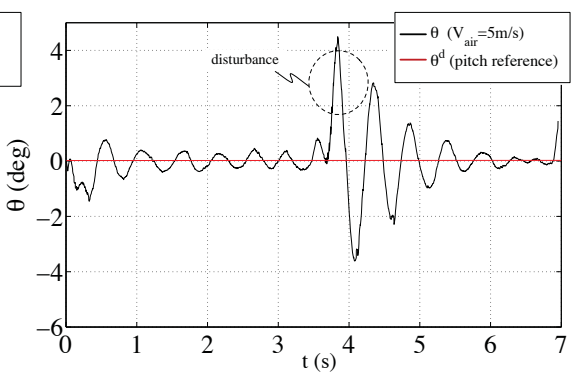

(b)

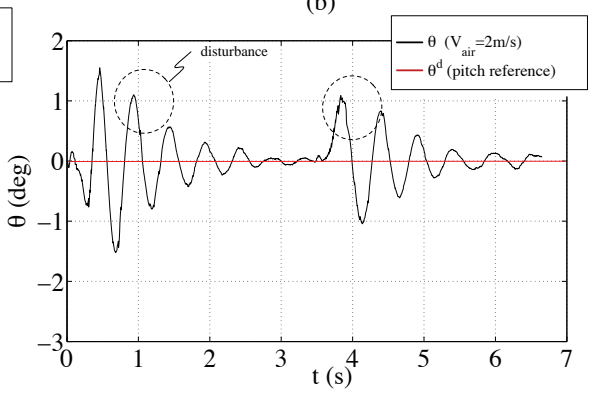

(d)

Figure 11. Turning flight control. Backstepping+DAF attitude tracking at: a)-b) roll and pitch tracking with $V_{\text {air }}=5 m s^{-1}$, c)-d) roll and pitch tracking with $V_{\text {air }}=2 m s^{-1}$.

\subsection{Forward and turning flight}

During forward flight, the bat-robot flaps the wings symmetrically at the desired wingbeat frequency $f$. The bio-inspired angular trajectories of the wings are shown in figure 7e-f-g. Wing modulation produces pitching and rolling torques $\left(\tau_{\phi}, \tau_{\theta}\right)$ due to wing inertias cause angular accelerations on the body. Using the inertial model of the robot, it is possible to determine both roll and pitch motions that are produced by these angular accelerations. The experiment carried out in figure 10 uses the calculation of roll and pitch motions as input references for the attitude controller $\left(\phi^{d}, \theta^{d}\right)$. The goal is to assess the accuracy of the backstepping+DAF method during the tracking of $\phi^{d}$ and $\theta^{d}$ when subjected to aerodynamic loads. As shown in figure 10a-b, the backstepping+DAF is able to maintain both $\phi, \theta$ oscillating around the defined set-point. The roll set-point is $0^{\circ}$ whereas the pitch set-point is $5.5^{\circ}$. This configuration allows for the generation of positive inertial thrust that would drive the robot forward. For this scenario the wind-tunnel airspeed has been set to $5 m s^{-1}$. Figures $10 \mathrm{c}-\mathrm{d}$ follow the same procedure with the difference that pitch set-point has been set to $20^{\circ}$

and the airspeed has been decreased to $2 \mathrm{~ms}^{-1}$. The insets show the attitude tracking errors caused by aerodynamic loads that depend on the airspeed.

In turning flight, the roll angle must be modified to allow the robot to turn right or left. Figure 11 shows experimental results on how the robot behaves during this process. Roll references are defined of the form $\phi^{d}=a+b \sin (2 \pi f t)$, where $a$ is the roll set-point angle, $b$ is the amplitude of the oscillation, and $f$ the desired frequency. On the other hand, pitch references have been set to $\theta^{d}=0^{o}$. In figure $11 \mathrm{a}$, the roll reference $\phi^{d}=a+0.25 \sin (2 \pi f t)$ determines that the robot must turn from right to left by following the set-point command: 

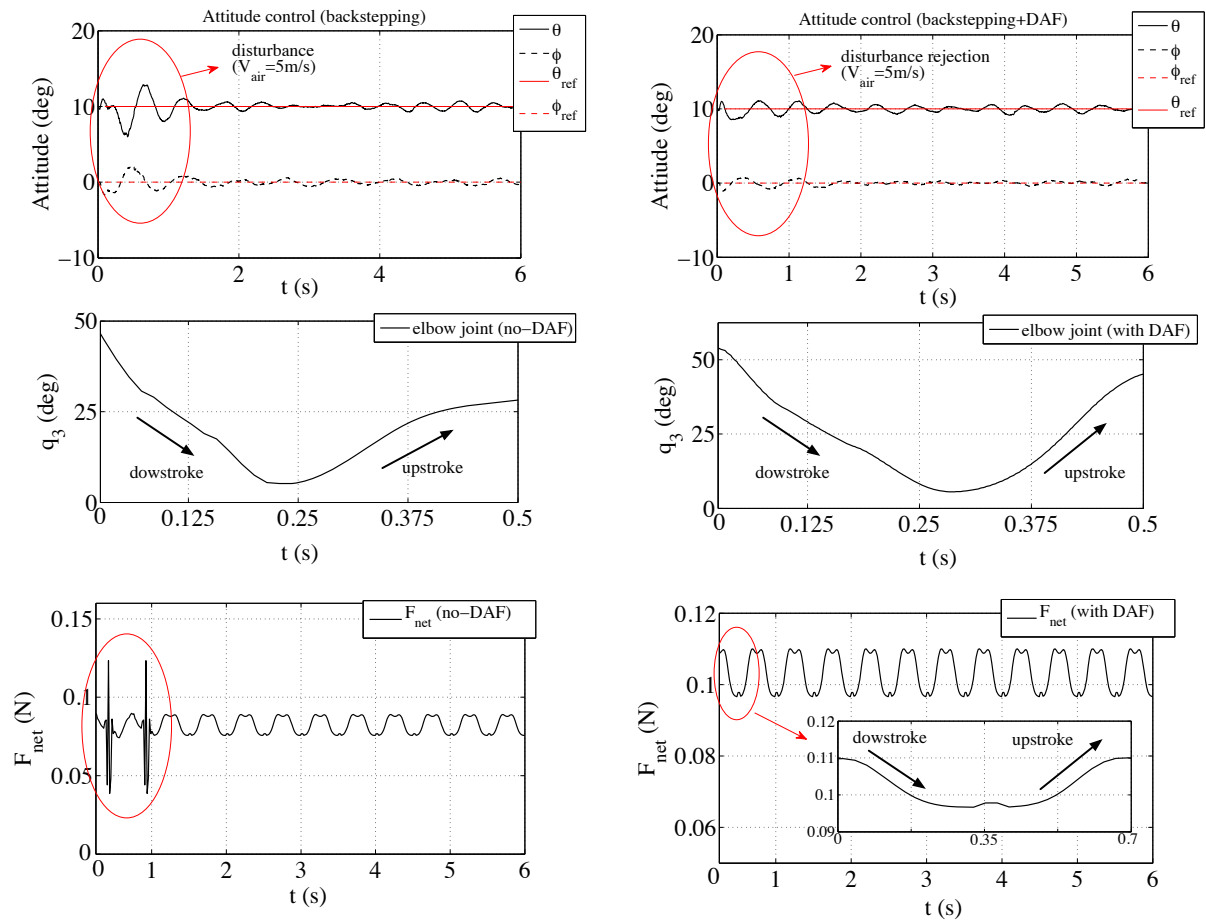

(a)

(b)

Figure 12. (Forward flight) benefits of the DAF to the proper modulation of wingmorphology aimed at incrementing net forces $\left(f=2 \mathrm{~Hz}, \phi_{\text {ref }}=0^{\circ}, \theta_{\text {ref }}=10^{\circ}\right)$ : a) without the DAF, b) with the DAF. Top: attitude tracking error and disturbance rejection; middle: detailed wing modulation (elbow joint $q_{3}$ ); bottom: net forces generated.

$a=7^{\circ}(0<t \leq 4)$, and $a=-2.5^{\circ}(4<t \leq 7)$. Disturbances have been induced into the system aimed at testing the reliability of the controller. These disturbances are caused by small loss of lift forces that occur during the contraction process of the wing. This issue can be observed in Figs. 11a-c at the switching point when $t=4 \mathrm{~s}$. The loss in lift forces accounted for about $4 \%$ due to a loss of tension of the wing membrane. In this work the loss of tension has not been quantified. This issue could be solved by improving the anisotropic property of the wing membrane material. Disturbances of this kind are difficult to immediately reject at high airspeeds $\left(5 m s^{-1}\right)$, however the backstepping+DAF controller has shown accurate performance in attenuating the amplitude of these oscillations at nominal airspeeds of $2 \mathrm{~ms}^{-1}$ (cf. figure 11c-d). Without the DAF, the oscillations caused by the disturbances would be higher than those observed here. Therefore, the following experiments are aimed at comparing the attitude response of the system with and without the DAF function, showing the benefits of the proposed controller in terms of disturbance rejection and net force production.

\subsection{Net force production}

Wing inertia information is contained in the DAF with the purpose of including desired roll and pitch angular accelerations within the control law. The DAF allows for the 
Table 6. List of parameters used for experiments in figure 12.

\begin{tabular}{ccccccccc}
\hline \hline Backstepping & AoA & $V_{\text {air }}$ & $f$ & $\bar{F}_{L}$ & $\bar{F}_{D}$ & $f_{z b}{ }^{1}$ & $f_{x b}$ & $F_{\text {net }}$ bias \\
\hline with DAF & $9^{\circ}$ & $5 m s^{-1}$ & $2 H z$ & $0 . \overline{9} 7 N$ & $0 . \overline{0} 99 N$ & $0.77 N$ & {$[-4,+9] m N$} & $0.11 N$ \\
without DAF & $9^{o}$ & $5 m s^{-1}$ & $2 H z$ & $0 . \overline{9} 7 N$ & $0 . \overline{1} 2 N$ & $0.77 N$ & {$[-4.5,+7.5] m N$} & $0.09 N$ \\
\hline \hline
\end{tabular}

${ }^{1} M_{b}=79 g$, no-battery included.

proper modulation of wing kinematics, which impacts the generation of both inertial and aerodynamic forces. Figure 12 quantifies the improvement in forward flight by carrying out measurements of: i) attitude tracking $(\phi, \theta)$, ii) wing modulation $\left(q_{3}\right)$, and iii) net force production $\left(F_{n e t}\right)$. Note how the backstepping+DAF is able to reject disturbances caused by increasing the wind-tunnel airspeed up to $5 \mathrm{~ms}^{-1}$ (top plots). Like the experiments in figure 10, accurate roll and pitch tracking is ensured during forward and turning flight. Each controller (with and without DAF) produce a different pattern of wing modulation $\left(q_{3}\right)$ (middle plots). With DAF the upstroke portion of the wingbeat cycle generates less drag thanks to the fact that the elbow joint contracts sufficiently to reduce the wing area at minimum span. This clearly affects the value of $F_{n e t}$ and the inertial thrust components $\left(f_{x b}\right)$ in Eq. (5). Finally, note in the bottom plots how with DAF the bias of net forces $F_{n e t}$ is about $23 \%$ higher thanks to the proper modulation of the wing kinematics. Also, it is confirmed that inertial thrust is positive during the upstroke and negative during the downstroke, causing the net force to increase or decrease as a function of the wingstroke motion (see inset in figure 12b). Table 6 summarises the numerical data.

\section{Discussion and Conclusions}

The results presented in this paper demonstrate how the wings can considerably affect the dynamics/aerodynamics of flight and how to take advantage of wing inertia information to properly change wings' geometry during flapping. This fact has been carefully modelled and quantified for the prototype at hand. It has also been shown how an effective attitude control of the bat-like robot can be achieved by changing wings' kinematics in order to generate controlled inertial forces. Moreover, the proposed control strategy was developed and tested for demonstrating the assumption of incrementing net body forces thanks to the wing modulation driven by the backstepping+DAF controller. Such increment is significant, about $23 \%$.

The robot's body mass influence in the generation of pitching and rolling torques has been quantified, and scaling factors that relate how both inertial quantities increase as a function of the flapping frequency, were found. These factors allowed the tuning of the backstepping+DAF control parameters, improving the attitude tracking against high external disturbances produced by aerodynamic loads. The results presented in this paper can be useful for control design purposes in future morphing wings MAVS (not necessarily bat-like), which have an important wing-to-body mass ratio. 
Mimicking the way bats take advantage of inertial and aerodynamical forces produced by the wings in order to both increase lift and maneuver is a promising way to design more efficient flapping wings MAVs. This is a key factor for their effective use in practical applications where the extremely low payload capacity limits the autonomy operation of flying machines in outdoor scenarios. In order to increase the efficiency of the morphing wings, further investigations regarding the highly-anisotropic property of the wing membrane should be taken into account. Furthermore, future wing designs should incorporate additional sensors, such as a flex sensor bound to the membrane or skeleton. This will give realtime knowledge of the shape of the wing, allowing the wings' shape in response to external variables to be tailored.

The novel wing modulation strategy and attitude control methodology presented and validated herein provide a totally new way of controlling flying robots that eliminates the need of appendices such as flaps and rudders. This is a promising approach for developing Micro Aerial Vehicles capable of aggressive maneuvers, especially useful in confined spaces: the fastest maneuvers in flying animals could be reproduced in man made flying vehicles.

\section{Acknowledgment}

This work is funded by the ROBOCITY 2030-II, sponsored by the Community of Madrid. Measurements of aerodynamic and inertial forces have been carried out using the Brown University wind-tunnel facility. The authors would like to thank to professors Kenny Breuer and Sharon Swartz for providing the support and useful knowledge about the robot design, bat flight kinematics and aerodynamics.

\section{Bibliography}

[1] Gomez, J.C., and Garcia, E., "Morphing unmanned aerial vehicles", Smart Mater. Struct, vol. 20, no. 10, 2011, pp 1-16.

[2] Wickenheiser, A., and Ephrahim, G., "Optimization of Perching Maneuvers Through Vehicle Morphing”, Journal of Guidance, Control, and Dynamics, vol. 31, no. 4, 2008, pp. 815-823.

[3] Chakravarthy, A., Grant, D., and Lind, R., "Time-Varying Dynamics of a Micro Air Vehicle with VariableSweep Morphing", Journal of Guidance, Control, and Dynamics, Vol. 35, No. 3, 2012, pp. 890-903.

[4] Bilgen, O., Marqui, C., Kochersberger, K., and Inman, D., "Macro-fiber composite actuators for flow control of a variable camber airfoil", J. Intell. Mater. Syst. Struct., vol. 22, 2011, pp. 81-91.

[5] Strelec, J., Lagoudas, D., Khan, M., and Yen, J., "Design and implementation of a shape memory alloy actuated reconfigurable airfoil”, J. Intell. Mater. Syst. Struct., vol. 14, 2003, pp. 257-73.

[6] Popov, A., Grigorie, L., and Botez, R., "Closed-Loop Control Validation of a Morphing Wing Using Wind Tunnel Tests", J. Aircr., vol. 47, 2010, pp.1309-1317.

[7] Vos, R., DeBreuker, R., Barrett, R., and Tiso, P., "Morphing wing flight control via postbuckled precompressed piezoelectric actuators", J. Aircr., Vol. 44, 2007, pp.1060-9.

[8] Rakotomamonjy, T., Ouladsine, M., Le Moing, T., "Longitudinal modelling and control of a flapping-wing micro aerial vehicle", Control Engineering Practice, Vol. 18, 2010, pp.679-690.

[9] Doman, D.B., Michael, W., Oppenheimer, and Sigthorsson, D.O., "Wingbeat Shape Modulation for Flapping-Wing Micro-Air-Vehicle Control During Hover", Journal of Guidance, Control, and Dynamics, 2010 vol. 33, no. 3, pp. 724-739.

[10] Lentink. D., "Nature inspired flight-beyond the leap", Bioinsp. Biomim., vol. 5, no. 4, 2010 , p040201. 
[11] Lentink, D., Muller, U.K., Stamhuis, E.J., Kat, R., Gestel, W., Veldhuis, L.L.M., Henningsson, P., Hedenstrom, A., Videler, J.J., and Leeuwen, J.L., "How swifts control their glide performance with morphing wings", Nature Letters, vol. 446, 2007, pp.1082-1085.

[12] Riskin, D. K., Willis, D. J., Iriarte-Diaz, J., Hedrick, T. L., Kostandov, M., Chen, J., Laidlaw, D. H., Breuer, K. S. and Swartz, S. M. "Quantifying the complexity of bat wing kinematics", Journal of Theoretical Biology, vol. 254, 2008, pp. 604-615.

[13] Iriarte-Diaz, J., Riskin, D.K., Willis, D.J., Breuer, K.S., and Swartz, S.M., "Whole-body kinematics of a fruit bat reveal the influence of wing inertia on body accelerations", Journal of Experimental Biology, vol. 214, no. 9, pp. 1546-1553, 2011.

[14] Riskin, D.K., Iriarte-Diaz, J., Middleton, K.M., Breuer, K.S., and Swartz, S.M., "The effect of body size on the wing movements of pteropodid bats, with insights into thrust and lift production", Journal of Experimental Biology, vol. 213, no. 23, pp. 4110-4122, 2010

[15] Swartz, S. M., Groves, M. D., Kim, H. D. and Walsh, W. R, Mechanical properties of bat wing membrane skin. J. Zool. 239, 357-378, 1996.

[16] S. Sterbing-D'Angelo, M. Chadha, C. Chiu, B. Falk, W. Xian, J. Barcelo, J. M. Zook, and C. F. Moss, Bat wing sensors support fight control, Proceedings of the National Academy of Sciences, vol. 108, no. 27, pp. 11291-11296, Jul. 2011.

[17] Swartz, S.M., Galvao, R., Iriarte-Diaz, J., Israeli, E., Middleton, K., Roemer, R., Tian, X. and Breuer, K., "Unique characteristics of aerodynamics of bat flight evidence from direct visualization of patterns of airflow in the wakes of naturally flying bats", Integ. Comp. Biol., vol. 45, 2005, pp.1080.

[18] Hedenstrom, A., Johansson, L.C., and Spedding, G.R., "Bird or bat: comparing airframe design and flight performance". Bioinsp. Biomim., vol. 4, no.1 2009, pp. 5001.

[19] J. Colorado, A. Barrientos, C. Rossi, and K. Breuer. "Biomechanics of smart wings in a bat robot: morphing-wings using SMA actuators", Bioinsp. Biomim., vol. 7 (3) pp. 36, 2012.

[20] A. J. Bergou, S. Swartz, K. S. Breuer, G. Taubin. "3D Reconstruction and Analysis of Bat Flight Maneuvers from Sparse Multiple View Video", In Proceedings of the 1st IEEE Symposium on Biological Data Visualization, 2011.

[21] Tian, X., Iriarte-Diaz, J., Middleton, K., Galvao, R., Israeli, E., Roemer, A., Sullivan, A., Song, A., Swartz, $\mathrm{S}$. and Breuer, K, Direct measurements of the kinematics and dynamics of bat fight, Bioinspir. Biomim. 1, pp.10-18, 2006.

[22] Thollesson, M., and Norberg, U.M., "Moments of inertia of bat wings and body", Journal of Experimental Biology, vol. 158, 1991, pp. 19-35.

[23] Featherstone, R., "Rigid Body Dynamics Algorithms", 2008, Springer, New York.

[24] Watts, P., Mitchell, E.J., and Swartz, S.M.,"A computational model for estimating the mechanics of horizontal flapping flight in bats: model description and validation", Journal of Experimental Biology, vol. 204, 2001, pp. 2873-2898.

[25] Denavit J. and Hartenberg R.S. "A Kinematic Notation for Lower Pair Mechanisms Based on Matrices", Trans. ASME J. Applied Mechanics, vol. 22, pp. 215-221, 1955. 58.

[26] Phillips, W. F. "Mechanics of Flight". Hoboken, NJ: Wiley. 2004

[27] N. Ma, G. Song, and H.-J. Lee, "Position control of shape memory alloy actuators with internal electrical resistance feedback using neural networks", Smart Mater. Struct 13, pp. 777-783, 2004.

[28] N. T. Tai and K. K. Ahn, "Adaptive proportional-integral-derivative tuning sliding mode control for a shape memory alloy actuator", Smart Mater. Struct., 20, pp. 10, 2011.

[29] Y. H. Teh and R. Featherstone. "An Architecture for Fast and Accurate Control of Shape Memory Alloy Actuators. The International Journal of Robotics Research' 27, pp. 595-611, 2008.

[30] O. Harkegard. "Flight Control Design Using Backstepping", Ph.D. Dissertation, Department of Electrical Engineering, Linkopings universitet, Sweden, 2001.

[31] S. Bouabdallah. "Design and control of quadrotors with application to autonomous flying", Ph.D. Dissertation, Ecole Polytechnique Federale de Lausanne (EPFL), Switzerland, 2007.

[32] Iriarte-Diaz, J., and Swartz, S.M., (2008). "Kinematics of slow turn maneuvering in the fruit bat Cynopterus brachyotis", The Journal of Experimental Biology, vol. 211, no. 47, 2008, pp. 3478-3489. 